\title{
Perturbation Theory for Evaluation Algorithms of Arithmetic Expressions
}

\author{
By F. Stummel
}

\begin{abstract}
The paper presents the theoretical foundation of a forward error analysis of numerical algorithms under data perturbations, rounding error in arithmetic floating-point operations, and approximations in 'built-in' functions. The error analysis is based on the linearization method that has been proposed by many authors in various forms. Fundamental tools of the forward error analysis are systems of linear absolute and relative a priori and a posteriori error equations and associated condition numbers constituting optimal bounds of possible accumulated or total errors. Derivations, representations, and properties of these condition numbers are studied in detail. The condition numbers enable simple general, quantitative definitions of numerical stability, backward analysis, well- and ill-conditioning of a problem and an algorithm. The well-known illustration of algorithms and their linear error equations by graphs is extended to a method of deriving condition numbers and associated bounds. For many algorithms the associated condition numbers can be determined analytically a priori and be computed numerically a posteriori. The theoretical results of the paper have been applied to a series of concrete algorithms, including Gaussian elimination, and have proved to be very effective means of both a priori and a posteriori error analysis.
\end{abstract}

Introduction. Evaluation algorithms are defined by finite sequences $F=$ $\left(F_{0}, \ldots, F_{n}\right)$ of input operations, evaluations of 'built-in' functions, and arithmetic operations for determining sequences $u=\left(u_{0}, \ldots, u_{n}\right)$ of input data, intermediate and final results in the form

$$
u_{t}=F_{t}(u), \quad t=0, \ldots, n .
$$

It is presupposed in the following that $F_{0}$ is a constant function and the function values $F_{t}(x)$ depend on $x_{0}, \ldots, x_{t-1}$ but not on $x_{t}, \ldots, x_{n}$ for $t=1, \ldots, n$. Under perturbations, an evaluation algorithm yields approximations $v_{t}$ of $u_{t}$ that can be written in the form

$$
v_{t}=\left(1+e_{t}\right) F_{t}(v), \quad t=0, \ldots, n .
$$

The so-called local errors $e_{t}$ are the relative errors of the data input, function evaluation, or rounding in the arithmetic floating-point operation in step $t$ of the algorithm. We shall assume that the local errors are bounded by $\left|e_{t}\right| \leqslant \gamma_{t} \eta$ for $t=0, \ldots, n$, where $\gamma_{t}$ are suitable nonnegative weights and $\eta$ is an accuracy constant.

Received June 12, 1979; revised November 26, 1979 and August 27, 1980.

1980 Mathematics Subject Classification. Primary 65G05.

Key words and phrases. Rounding error analysis, evaluation algorithms, a priori and a posteriori error estimates, condition numbers, linear error equations, graphs. 
In vector notation, (1), (2) may be written $u=F(u), v=F(v)+d$, using the residual vector $d$ with the components $d_{t}=-v_{t} e_{t}^{\prime}$ and $e_{t}^{\prime}=-e_{t} /\left(1+e_{t}\right)$. By introducing the mapping $A=I-F,(1),(2)$ become

$$
A u=0, \quad A v=d \text {. }
$$

Thus, the error analysis of an evaluation algorithm is a perturbation theory of the functional equation $A u=0$ with respect to perturbations of the right-hand side. By Taylor's formula at the point $u$, the absolute a priori error $\Delta u=v-u$ satisfies the relation $\left(A^{\prime} u\right) \Delta u+R=d$, where $R$ is a remainder term of order $O\left(\|\Delta u\|^{2}\right)$. Neglecting terms of second order in $\|\Delta u\|$ and $\eta$ gives $\left(A^{\prime} u\right) \Delta u \doteq J_{u} e$, where $J_{u}=\operatorname{diag}\left(u_{0}, \ldots, u_{n}\right)$. In the a posteriori error analysis, one uses Taylor's formula at the point $v$ and obtains the relation $\left(A^{\prime} v\right) \Delta v \doteq J_{v} e^{\prime}$ for the error $\Delta v=u-v$. The solutions $s$ of the associated systems of linear error equations

$$
\left(A^{\prime} u\right) s=J_{u} e \text {, or }\left(A^{\prime} v\right) s=J_{v} e^{\prime},
$$

then yield approximations of the absolute errors $\Delta u$ or $\Delta v$. Correspondingly, approximations $r$ of the relative errors $P u=J_{u}^{-1} \Delta u$ or $P v=J_{v}^{-1} \Delta v$ are obtained from the systems of linear error equations

$$
J_{u}^{-1}\left(A^{\prime} u\right) J_{u} r=e, \text { or } J_{v}^{-1}\left(A^{\prime} v\right) J_{v} r=e^{\prime} .
$$

The procedure, described above, is analogous to the derivation of difference approximations of a differential equation; the role of truncation errors is played by remainder terms $O\left(\|\Delta u\|^{2}\right), O\left(\eta^{2}\right)$ here. Note that the linear error equations can also be derived simply from the exact error equations in Section 1.1 by neglecting terms of second order in the errors and replacing the absolute and relative errors of $u, v$ by $s$ and $r$.

The subject of the paper is a study of the structure of the mapping $A$ and its Fréchet-derivative $A^{\prime} w$, the derivation and proof of error representations of the form

$$
\Delta w_{t}=s_{t}+O_{t}\left(\eta^{2}\right), \quad P w_{t}=r_{t}+O_{t}\left(\eta^{2}\right),
$$

where $s, r$ are the solutions of (4), (5), and associated optimal error estimates

$$
\left|\Delta w_{t}\right| \leqslant \sigma_{t} \eta+O_{t}\left(\eta^{2}\right), \quad\left|P w_{t}\right| \leqslant \rho_{t} \eta+O_{t}\left(\eta^{2}\right),
$$

a detailed analysis of the remainder terms in (6), (7) and of the properties of the optimal constants $\sigma_{t}, \rho_{t}$. In (6), (7), one chooses $w=u$ in an a priori and $w=v$ in an a posteriori error analysis. The components $s_{t}, r_{t}$ of $s, r$ are linear forms in the local errors $e=\left(e_{0}, \ldots, e_{n}\right)$, and the bounds $\sigma_{t} \eta, \rho_{t} \eta$ are associated norms, specified by the error distribution, $\left|e_{t}\right| \leqslant \gamma_{t} \eta$, of these linear forms. The constants $\sigma_{t}, \rho_{t}$ are the weighted absolute and relative, a priori and a posteriori condition numbers.

Particular attention is paid in the following to the simultaneous treatment of absolute and relative, a priori and a posteriori errors as well as to readily accessible presentations of the coefficients and inhomogeneous terms of the associated error equations, of condition numbers, weights, and so on, in all four cases. For, in applications to concrete examples it is seen that for some algorithms the systems of linear absolute error equations, for others the systems of linear relative error equations, are easier to handle. The general theoretical rounding error analysis of 
numerical algorithms uses a priori error representations. In many examples, condition numbers can be computed numerically a posteriori, that is, together with the intermediate and final results of the algorithm.

By a suitable choice of the weights in the local error distribution, the behavior of the algorithm can be studied under data perturbations only, assuming exact 'built-in' functions and arithmetic operations, as well as under rounding errors in the arithmetic operations and 'built-in' functions only, assuming exact data. The associated absolute and relative condition numbers are denoted by $\sigma_{t}{ }^{D}, \sigma_{t}^{R}$ and $\rho_{t}^{D}$, $\rho_{t}{ }^{R}$. The data condition numbers $\sigma_{t}^{D}$ are absolute asymptotic conditions, in the sense of Rice [15], of $u_{t}$ viewed as a function of the data and thus independent of the algorithm (see Section 2.1). By Wilkinson [25, I.36], a problem is said to be ill-conditioned if small relative errors of the input data can induce great relative errors of the solution $u_{t}$ of the algorithm. This effect can be formulated quantitatively in our terms by $\rho_{t}^{D} \gg 1$. Wilkinson's backward analysis describes the behavior of the algorithm under rounding errors in the arithmetic operations by data perturbations. Our results in Section 2.1 show that $\sigma_{t}^{R} / \sigma_{t}^{D}=\rho_{t}^{R} / \rho_{t}^{D}$ is a measure for the magnitude of those data perturbations which are necessary and sufficient in order to represent perturbations by rounding errors in the arithmetic operations. Bauer's concept (see [4], [5]) of a well-conditioned algorithm for the computation of $u_{t}$ can be specified by the requirement that $\rho_{t}^{R} / \rho_{t}^{D}$ is not much greater than one.

Also, a well-conditioned algorithm can produce numerical results such that not even the first digit is significant. This happens when the problem is ill-conditioned and the accuracy $\eta$ is too low. The least number of significant digits in a computed result can be determined by our relative condition numbers $\rho_{t}$ and the associated notion of numerical stability. Let data perturbations and rounding errors, within a given distribution of local errors, be bounded by the accuracy $\eta_{0}$. Then the result $v_{t}$ is computed under all these perturbations within the relative error bound $\eta_{1}$ if the stability inequality $\rho_{t}<\eta_{1} / \eta_{0}$ holds. This result follows immediately from (7) for $\eta=\eta_{0}$. For example, choosing $\eta_{1}=\frac{1}{2}$ and $\eta_{0}<1 /\left(2 \rho_{t}\right)$ guarantees that at least the first digit in the computed result $v_{t}$ is accurate in the sense of relative errors. It is always assumed that remainder terms of the form $O\left(\eta^{2}\right)$ are negligible against the terms of first order in $\eta$.

Evaluation algorithms and their systems of linear error equations may be illustrated by graphs constituting detailed complete flow diagrams of the functional dependences between the data, intermediate and final results, as well as exhibiting the paths of error propagation and the associated error effects along these paths. It seems that this tool is found for the first time in the book of McCracken-Dorn [11] who attribute the idea to H. M. King. In essentially the same form Bauer [5], Brown [7], Linnainmaa [10], and others use graphs in their error analyses. The matrix elements of the solution operators $\left(A^{\prime} w\right)^{-1}, J_{w}^{-1}\left(A^{\prime} w\right)^{-1} J_{w}$ can be read from the weighted graph. In addition, Section 2.3 provides graph theoretic means for determining condition numbers and associated bounds. For instance, when the graph of the algorithm is a tree, condition numbers are obtained recurrently by simple formulae having the same form as those of the condition numbers of the simplest algorithms in Section 1.1. 
Note that the concepts of condition numbers used, for example, by Wilkinson [25], Bauer [5], or the well-known condition numbers of matrices, are defined differently and have other meanings. In the rounding error analysis of a fixed-point arithmetic, Henrici $[8,16.4]$ states a system of linear equations for the exact errors $\Delta u_{t}$. Also the optimality of error bounds, obtained in this way, is observed there. Linnainmaa [10] studies Taylor expansions of the total errors $\Delta u_{t}$ with respect to the local errors $e_{0}, \ldots, e_{n}$. In particular, two algorithms for computing the coefficient matrices of the terms of first and second order in the expansions of the total errors are presented. We can interpret the first algorithm as a procedure to compute the rows of the inverse matrix $\left(A^{\prime} u\right)^{-1}$ from the matrix $F^{\prime}(u)$ (see Section 1.3). This algorithm has been described by Larson-Sameh [9] in a somewhat different context. Bauer [5], Brown [7], and Larson-Sameh [9] use relative or logarithmic derivatives for obtaining representations of the total relative errors $P u_{t}$. This method yields first-order approximations of the errors that coincide with the solutions $r_{t}$ of the system of linear relative a priori error equations (5).

Another way of deriving a priori error representations and estimates of the kind described above starts from an analysis of the perturbed results $v_{t}$ viewed as functions of the local errors $e=\left(e_{0}, \ldots, e_{n}\right)$. Let $v_{t}=G_{t}(e)$, then $u_{t}=G_{t}(0)$ and, by Taylor expansion,

$$
\Delta u_{t}=G_{t}^{\prime}(0) e+O_{t}\left(\eta^{2}\right) .
$$

From (4), (6) with $w=u$, and (8) it follows that $s_{t}=G_{t}^{\prime}(0) e$ for all local errors $e$ and

$$
G_{t}^{\prime}(0)=\operatorname{pr}_{t}\left(A^{\prime} u\right)^{-1} J_{u}
$$

where $\mathrm{pr}_{t}$ denotes the orthogonal projection onto the $t$ th coordinate axis. The absolute a priori condition numbers thus permit the representation

$$
\sigma_{t}=\lim _{\eta \rightarrow+0} \frac{1}{\eta} \sup _{|e|<\gamma \eta}\left|G_{t}(e)-G_{t}(0)\right|=\sum_{k=0}^{t}\left|\frac{\partial G_{t}}{\partial e_{k}}(0)\right| \gamma_{k} .
$$

Babuška's stability constants $\Lambda$ in [1], [2] are defined in the form of the first equation in (10). Miller's papers [12], [13] use condition numbers of the kind defined by the second equation in (10). The associated stability constants $1+$ $\sigma_{t}^{R} / \sigma_{t}^{D}$ are denoted by $\xi$ in [1], [2]. Miller [12], [13] and Miller-Spooner [14] use the notation $\rho$ and $\omega_{1}$ for $\sigma_{t}^{R} / \sigma_{t}^{D}$. When the error analysis is applied to concrete algorithms, the functional dependence $G_{t}$ of $v_{t}$ on the local errors is, in general, very complex, so that this approach is limited to small algorithms.

Although the total number of operations, and thus the order of the associated matrices $A^{\prime} w$, becomes very large for many important algorithms, the matrices $A^{\prime} w$ are very sparse and highly structured. The directed graph of the functional dependences of an algorithm and the directed graph of an associated linear system of error equations are identical. Thus the structure of the system of linear error equations is, necessarily, related to that of the algorithm. This fact might be the deeper reason why for many important algorithms, including Gaussian elimination of systems of linear algebraic equations, explicit analytical representations of the solutions of the systems of linear error equations and of the associated condition numbers can be determined. 
In a series of papers, surveyed in Section 3, the present error analysis has been applied to concrete numerical algorithms. The error estimates have been tested by numerous numerical examples. All these examples have confirmed that the condition numbers clearly and concisely yield crucial information about the numerical behavior of the algorithms. The a posteriori condition numbers have proved to be reliable measures of the magnitude of possible errors.

1. Elements of Error Propagation. The first chapter introduces basic concepts of the perturbation theory for numerical algorithms. Starting points are representations and estimates of the errors in elementary arithmetic operations,,,$+- \times, /$, and 'built-in' functions occurring in the floating-point arithmetic of computers. In particular, condition numbers are defined for the simplest algorithms, consisting of the input of one or two operands followed by an arithmetic operation or function evaluation. It will be seen in Section 2.3 that these condition numbers are also used in determining condition numbers or associated bounds for general algorithms. In Section 1.2 the class of algorithms for evaluating arithmetic expressions is defined and the general form of perturbed algorithms specified in view of typical rounding error analyses. Further, the general error equations for the solutions of the perturbed algorithms are derived, and associated estimates for the remainder terms of Taylor's formula are established. By neglecting remainder terms in the general error equations, linear error equations arise whose solutions approximate the absolute and relative a priori and a posteriori errors being of interest. The algorithm (A) determines uniquely a mapping $A$ in $\mathbf{R}^{n+1}$. It will be shown in Section 1.3 that the linear error equations are defined by the Fréchet-derivative $A^{\prime}$ of $A$. The solutions of the linear absolute error equations are obtained by means of the solution operators $L=\left(A^{\prime} w\right)^{-1}$, and of the relative error equations by $L=$ $J_{w}^{-1}\left(A^{\prime} w\right)^{-1} J_{w}$. This is in correspondence with the use of so-called relative or logarithmic derivatives (see Bauer [5], Brown [7]).

1.1. Error Estimates for the Simplest Numerical Algorithms. Given two numbers $a$, $b$ and arbitrary approximations $a^{\prime}, b^{\prime}$ of $a, b$, the following absolute and relative $a$ priori and $a$ posteriori errors are defined:

\begin{tabular}{|l|l|l} 
& absolute & relative \\
\hline a priori & $\Delta a=a^{\prime}-a$ & $P a=\frac{a^{\prime}-a}{a}$ \\
\hline a posteriori & $\Delta a^{\prime}=a-a^{\prime}$ & $P a^{\prime}=\frac{a-a^{\prime}}{a^{\prime}}$ \\
\hline
\end{tabular}

In the relative error analysis it will always be assumed that denominators are different from zero. Let us first state representations of the absolute and relative a priori errors

(2) $\Delta(a \circ b)=a^{\prime} \circ b^{\prime}-a \circ b, \quad P(a \circ b)=\frac{a^{\prime} \circ b^{\prime}-a \circ b}{a \circ b}, \quad \circ=+,-, \times, /$, of sums, differences, products, and quotients under perturbations of the operands $a, b$. It is well known that 


$$
\begin{array}{ll}
\Delta(a \pm b)=\Delta a \pm \Delta b, & P(a \pm b)=\frac{a}{c} P a \pm \frac{b}{c} P b, \\
\Delta(a b)=b \Delta a+a \Delta b+\Delta a \Delta b, & P(a b)=P a+P b+P a P b, \\
\Delta(a / b)=\frac{1}{b+\Delta b}\left(\Delta a-\frac{a}{b} \Delta b\right), & P(a / b)=\frac{P a-P b}{1+P b},
\end{array}
$$

where $c=a \pm b$. The numerical computation of $a \circ b$ first requires input or computing of the operands $a, b$ carried out, in general, approximately. The arithmetic floating-point operations are then applied to approximations of $a, b$. Input of $a, b$ gives, for instance, $a^{\prime}=\mathrm{fl}(a), b^{\prime}=\mathrm{fl}(b)$. By $\mathrm{fl}$ is meant a function mapping the real numbers into $N$-digit floating-point numbers. Let $g$ denote the base of the number representation. The floating-point arithmetic thus computes the approximations

$$
v=\mathrm{fl}\left(a^{\prime} \circ b^{\prime}\right)=(1+e)\left(a^{\prime} \circ b^{\prime}\right), \quad|e| \leqslant \eta,
$$

of the exact results $u=a \circ b$ where $\circ=+,-, \times, /$ (see Wilkinson [25]). The floating-point accuracy constant $\eta$ is, for example, $\frac{1}{2} g^{-N+1}$ when $\mathrm{fl}$ is symmetric rounding, and $g^{-N+1}$ when $\mathrm{fl}$ denotes chopping off the mantissae to $N$ digits. Now

$$
\Delta u=v-u=(1+e)\left(a^{\prime} \circ b^{\prime}-a \circ b\right)+e(a \circ b),
$$

so that the absolute and relative a priori errors of $v$ have the representations

$$
\Delta u=(1+e) \Delta(a \circ b)+u e, \quad P u=(1+e) P(a \circ b)+e .
$$

Inserting the above expressions for $\Delta(a \circ b), P(a \circ b)$ in (3) yields the absolute and relative a priori error equations.

Analogous representations hold for the absolute and relative a posteriori errors

$$
\begin{aligned}
& \Delta\left(a^{\prime} \circ b^{\prime}\right)=a \circ b-a^{\prime} \circ b^{\prime}, \\
& P\left(a^{\prime} \circ b^{\prime}\right)=\frac{a \circ b-a^{\prime} \circ b^{\prime}}{a^{\prime} \circ b^{\prime}}, \quad \circ=+,-, \times, / .
\end{aligned}
$$

They are obtained by interchanging $a, b$ and $a^{\prime}, b^{\prime}$ in (3). Let us further introduce the notation

$$
e^{\prime}=-\frac{e}{1+e} \quad(e \neq-1)
$$

Then, evidently,

$$
e+e^{\prime}+e e^{\prime}=0, \quad(1+e)\left(1+e^{\prime}\right)=1 .
$$

Using (4), one has

$$
\Delta v=u-v=a \circ b-a^{\prime} \circ b^{\prime}-e\left(a^{\prime} \circ b^{\prime}\right), \quad a^{\prime} \circ b^{\prime}=\frac{v}{1+e} .
$$

The absolute and relative a posteriori errors of the approximation $v$ of $u$ can therefore be written in the form

$$
\Delta v=\Delta\left(a^{\prime} \circ b^{\prime}\right)+v e^{\prime}, \quad P v=\left(1+e^{\prime}\right) P\left(a^{\prime} \circ b^{\prime}\right)+e^{\prime} .
$$

From (3), (9) we obtain absolute and relative a posteriori error equations. Note that the representation of relative a priori errors turns into that of relative a posteriori errors when $a, b, e, u$ are interchanged by $a^{\prime}, b^{\prime}, e^{\prime}, v$. 
Next let a real function $f$ of a single variable be given, twice continuously differentiable in its open domain of definition. Under perturbations of the argument $a$, the induced approximations $\mathrm{f}\left(a^{\prime}\right)$ of $f(a)$ have the absolute and relative a priori errors

$$
\begin{aligned}
& \Delta f(a)=\left(1+\frac{f\left[a, a, a^{\prime}\right]}{f^{\prime}(a)} \Delta a\right) f^{\prime}(a) \Delta a, \\
& P f(a)=\left(1+\frac{a f\left[a, a, a^{\prime}\right]}{f^{\prime}(a)} P a\right) \frac{a f^{\prime}(a)}{f(a)} P a,
\end{aligned}
$$

using the Hermite generalized divided differences

$$
f\left[a, a, a^{\prime}\right]=\int_{0}^{1}\left(\int_{0}^{t} f^{\prime \prime}\left(a+s\left(a^{\prime}-a\right)\right) d s\right) d t .
$$

It is presupposed that $f^{\prime}(a)$ and, in the case of relative errors, $a$ and $f(a)$ are different from zero. The above representations are valid for all pairs $a, a^{\prime}$ such that the line segment $\overline{a a^{\prime}}$ belongs to the domain of definition of $f$.

In the numerical evaluation of real functions, instead of $u=f(a)$ an approximation of $f$ at a neighboring point $a^{\prime}$ of $a$ is computed. Let $v$ denote this approximation. Then

$$
v=(1+e) f\left(a^{\prime}\right), \quad e=\frac{v-f\left(a^{\prime}\right)}{f\left(a^{\prime}\right)} \quad\left(f\left(a^{\prime}\right) \neq 0\right) .
$$

It can be assumed, for example, that elementary 'built-in' functions are computed by

$$
v=\mathrm{fl}\left(f\left(a^{\prime}\right)\right) \text {. }
$$

In this case,

$$
e=\frac{\mathrm{fl}(y)-y}{y}, \quad y=f\left(a^{\prime}\right), \quad|e| \leqslant \eta .
$$

In line with (5), (9), one finds the error equations

$$
\begin{array}{ll}
\Delta u=(1+e) \Delta f(a)+u e, & P u=(1+e) P f(a)+e, \\
\Delta v=\Delta f\left(a^{\prime}\right)+v e^{\prime}, & P v=\left(1+e^{\prime}\right) P f\left(a^{\prime}\right)+e^{\prime},
\end{array}
$$

for the approximations $v$ of $u=f(a)$, where $e$ is specified by (12) and herewith $e^{\prime}$ by (7). Inserting the representations (10) of $\Delta f(a)$ and $P f(a)$ into (15) leads to the absolute and relative a priori error equations of numerical evaluations of $f$. Interchanging $a, a^{\prime}$ in (10) and inserting the resulting representations of $\Delta f\left(a^{\prime}\right), P f\left(a^{\prime}\right)$ into (15) yields the associated absolute and relative a posteriori error equations. Again, the relative a priori error equations change to the a posteriori error equations, and vice versa, when $a, e, u$ are interchanged by $a^{\prime}, e^{\prime}, u^{\prime}$.

The simplest numerical algorithms consist of the input of $a$ or $a, b$ followed by the computation of $f(a)$ or $a \circ b$ for $\circ=+,-, \times, /$. From the above error equations we can readily deduce associated error estimates. For this purpose, let us assume that the relative errors $P a, P b, e_{+}, e_{-}, \ldots$ of the input data and arithmetic floating-point operations,,$+- \ldots$ are bounded by

$$
|P a| \leqslant \rho_{a} \eta, \quad|P b| \leqslant \rho_{b} \eta, \quad|e| \leqslant \gamma \eta .
$$


The absolute errors $\Delta a, \Delta b$ then satisfy the estimates

$$
|\Delta a| \leqslant \sigma_{a} \eta, \quad|\Delta b| \leqslant \sigma_{b} \eta, \quad \sigma_{a}=|a| \rho_{a}, \quad \sigma_{b}=|b| \rho_{b} .
$$

The constants $\rho_{a}, \rho_{b}$ and $\sigma_{a}, \sigma_{b}$ are the relative and absolute a priori condition numbers of the data $a, b$. They permit adjusting the bounds to the magnitude of possible errors of the data approximations $a^{\prime}, b^{\prime}$. When $a, b$ are results of a previous numerical computation, in general $a^{\prime}, b^{\prime}$ have larger errors than input errors of magnitude $\eta$. Constants $\rho_{a}, \rho_{b}, \sigma_{a}, \sigma_{b}$ may be zero when input data are exact or unperturbed. The weight $\gamma$ can be set equal to zero if the floating-point operation is performed exactly, for instance, when a loss of significant figures occurs in subtractions. The error equations (3), (5), (10), (15) entail the absolute and relative a priori error estimates

$$
|\Delta u| \leqslant(1+O(\eta)) \sigma \eta, \quad|P u| \leqslant(1+O(\eta)) \rho \eta,
$$

using the following absolute and relative a priori condition numbers of arithmetic operations and function evaluations:

$$
\begin{aligned}
+,-: & \sigma & =\sigma_{a}+\sigma_{b}+|u| \gamma, & & =\left|\frac{a}{u}\right| \rho_{a}+\left|\frac{b}{u}\right| \rho_{b}+\gamma, \\
\times: & \sigma & =|b| \sigma_{a}+|a| \sigma_{b}+|u| \gamma, & & =\rho_{a}+\rho_{b}+\gamma, \\
/: & \sigma & =\frac{1}{|b|} \sigma_{a}+\left|\frac{u}{b}\right| \sigma_{b}+|u| \gamma, & & =\rho_{a}+\rho_{b}+\gamma, \\
f: & \sigma & =\left|f^{\prime}(a)\right| \sigma_{a}+|u| \gamma, & \rho & =\left|\frac{a f^{\prime}(a)}{f(a)}\right| \rho_{a}+\gamma .
\end{aligned}
$$

Note that always $\rho=\sigma /|u|, u=a \circ b$ for $\circ=+,-, \times, /$ or $u=f(a)$. The above estimates require for divisions that $\rho_{b} \eta<\vartheta<1$, and for function evaluations that $\left|f\left[a, a, a^{\prime}\right]\right| /\left|f^{\prime}(a)\right| \leqslant \mu$.

Let us assume that the a posteriori errors $P a^{\prime}, P b^{\prime}, e^{\prime}$ and $\Delta a^{\prime}, \Delta b^{\prime}$ satisfy estimates corresponding to (16), (17). From (3), (6), (9), (10), (15) the absolute and relative a posteriori error estimates

$$
|\Delta v| \leqslant(1+O(\eta)) \sigma \eta, \quad|P v| \leqslant(1+O(\eta)) \rho \eta
$$

follow, where $\sigma, \rho$ denote the absolute and relative a posteriori condition numbers

$$
\begin{aligned}
+,-: & \sigma & =\sigma_{a}+\sigma_{b}+|v| \gamma, & & \rho=\left|\frac{a^{\prime}}{v}\right| \rho_{a}+\left|\frac{b^{\prime}}{v}\right| \rho_{b}+\gamma, \\
\times: & \sigma & =\left|b^{\prime}\right| \sigma_{a}+\left|a^{\prime}\right| \sigma_{b}+|v| \gamma, & & =\rho_{a}+\rho_{b}+\gamma, \\
/: & \sigma & =\frac{1}{\left|b^{\prime}\right|} \sigma_{a}+\left|\frac{v}{b^{\prime}}\right| \sigma_{b}+|v| \gamma, & \rho & =\rho_{a}+\rho_{b}+\gamma, \\
f: & \sigma & =\left|f^{\prime}\left(a^{\prime}\right)\right| \sigma_{a}+|v| \gamma, & \rho & =\left|\frac{a^{\prime} f^{\prime}\left(a^{\prime}\right)}{f\left(a^{\prime}\right)}\right| \rho_{a}+\gamma .
\end{aligned}
$$

Now $\rho=(1+O(\eta)) \sigma /|v|, v=\mathrm{fl}\left(a^{\prime} \circ b^{\prime}\right)$ or $v=\mathrm{fl}\left(f\left(a^{\prime}\right)\right)$. It is always assumed in the present paper that $\eta$ and $O(\eta)$ are small compared to 1 . The quantities $a^{\prime}, b^{\prime}, v$ are floating-point numbers, so that a posteriori condition numbers can be computed numerically together with the result $v$.

The above estimates (18) are sharp or optimal for the class of all perturbations defined by (16). When $\rho_{a}, \rho_{b}, \gamma$ and the data $a, b$ are so chosen that

$$
P a=\operatorname{sgn}\left(\frac{a}{u}\right) \rho_{a} \eta, \quad P b=\operatorname{sgn}\left(\frac{b}{u}\right) \rho_{b} \eta, \quad e=\gamma \eta,
$$


the relative a priori error of the sum $u=a+b$ becomes

$$
P u=(1+\gamma \eta)\left(\left|\frac{a}{u}\right| \rho_{a}+\left|\frac{b}{u}\right| \rho_{b}\right) \eta+\gamma \eta,
$$

that is, $\rho \eta \leqslant P u \leqslant(1+\gamma \eta) \rho \eta$. Analogous statements are true for the other arithmetic operations and function evaluations, as well as for a posteriori error estimates.

1.2. Evaluation Algorithms and General Representations of Errors. The evaluation of arithmetic expressions by a computer is carried out in a series of elementary steps: input of numbers, arithmetic operations,,$+- \times, /$ and evaluations of elementary 'built-in' functions $V, \ln$, exp, sin, $\cos , \ldots$. In this section a class of finite algorithms for evaluating general arithmetic expressions is defined analogously to typical computer programs. As a rule, data have to be read into the storage first. From these data a sequence of intermediate and final results is computed stepwise. In doing so, further data may be read in. We assume that data, intermediate, and final results are stored in places having the addresses $0,1,2, \ldots, n$. In each step of the computation all previously computed results are available for further computations. An evaluation algorithm is thus defined by a constant function $F_{0}$, representing the input of a number, and a finite sequence of real functions $F_{t}$, having suitable domains of definition def $F_{t}$ in $\mathbf{R}^{t}$, in the form

$$
u_{0}=F_{0}, \quad u_{t}=F_{t}\left(u_{0}, \ldots, u_{t-1}\right), \quad t=1, \ldots, n .
$$

The functions $F_{t}$ specify how the next value is computed from the data and intermediate results $u_{0}, \ldots, u_{t-1}$ in storage places $0, \ldots, t-1$. In view of the further study it is expedient to use vector notation such that

$$
u=\left(u_{0}, \ldots, u_{n}\right), \quad v=\left(v_{0}, \ldots, v_{n}\right), \quad x=\left(x_{0}, \ldots, x_{n}\right), \ldots \in \mathbf{R}^{n+1} .
$$

The functions $F_{t}$ are then defined by

$$
F_{0}(x)=F_{0}, \quad F_{t}(x)=F_{t}\left(x_{0}, \ldots, x_{t-1}\right), \quad t=1, \ldots, n,
$$

for all $x \in \mathbf{R}^{n+1}$ with the property that $\left(x_{0}, \ldots, x_{t-1}\right)$ belongs to def $F_{t}$.

The class (F) of admissible functions $F_{t}$ is composed of three subclasses (F0), (F1), (F2) by

$$
F_{t} \in(\mathrm{F})=(\mathrm{F} 0) \cup(\mathrm{F} 1) \cup(\mathrm{F} 2), \quad t=0, \ldots, n .
$$

(F0) Input operations are represented by constant functions $F_{t}$ on all of $\mathbf{R}^{n+1}$. In the floating-point arithmetic of a computer input operations are carried out in the form

$$
\mathrm{fl}\left(F_{t}(x)\right)=\left(1+e_{t}\right) F_{t}(x) .
$$

The relative errors $e_{t}$ of these approximations are bounded, for instance, by $\left|e_{t}\right| \leqslant \eta$, where $\eta$ denotes the floating-point accuracy constant defined in Section 1.1 .

(F1) 'Built-in' functions. Functions in this class are defined by

$$
F_{t}(x)=f_{t}\left(x_{j_{t}}\right)
$$

using indices $j_{t} \in\{0, \ldots, t-1\}$ and elementary, 'built-in', functions

$$
f_{t} \in\{\vee, \ln , \exp , \sin , \cos , \ldots\} \text {. }
$$


The domain of definition of $F_{t}$ consists of all those $x$ in $\mathrm{R}^{n+1}$ such that $x_{j_{t}}$ belongs to def $f_{t}$. In floating-point arithmetic these functions are, at best, evaluated by

$$
\mathrm{fl}\left(F_{t}(x)\right)=\left(1+e_{t}\right) F_{t}(x), \quad\left|e_{t}\right| \leqslant \eta \text {. }
$$

In this class also functions $f_{t}$ of the form $f_{t}(z)=z \circ p_{t}$ are admitted, where $\circ=+,-, \times, /$ or $\uparrow$ and $p_{t}$ is a constant which can be represented exactly as a floating-point number. A simple example is $f_{t}(z)=z \uparrow 2$.

(F2) Arithmetic operations are represented by functions $F_{t}$ of the form

$$
F_{t}(x)=\left( \pm x_{i_{t}}\right) \circ\left( \pm x_{j_{t}}\right)
$$

where $i_{t}, j_{t}$ are distinct indices in $\{0, \ldots, t-1\}$, and $\circ$ are operations in $\{+,-, \times, /\}$. The domain of definition of $F_{t}=+,-, \times$ is all of $\mathbf{R}^{n+1}$. In the case of divisions, def $F_{t}=\left\{x \in \mathbf{R}^{n+1} \mid x_{j_{t}} \neq 0\right\}$. Let us assume that the floating-point arithmetic of our computer evaluates the arithmetic operations approximately by

$$
\mathrm{fl}\left(F_{t}(x)\right)=\left(1+e_{t}\right) F_{t}(x), \quad\left|e_{t}\right| \leqslant \eta \text {. }
$$

Note that $-x_{i_{1}}$ is obtained exactly from $x_{i}$ by a change of sign.

The evaluation of functions $F_{t} \in(\mathrm{F})$ in the floating-point arithmetic of a computer is carried out approximately as described above. Instead of the sequence of solutions $u_{0}, \ldots, u_{n}$ of (A), a sequence of approximations $v_{0}, \ldots, v_{n}$ is computed such that $v_{t}$ is an approximation of $y_{t}=F_{t}(v)$ for each $t$. Let $e_{t}$ denote the relative a priori error of the approximation $v_{t}$ of $y_{t}$ and let $e_{t}^{\prime}$ be the associated relative a posteriori error,

$$
e_{t}=\frac{v_{t}-y_{t}}{y_{t}}=P y_{t}, \quad e_{t}^{\prime}=-\frac{e_{t}}{1+e_{t}}=\frac{y_{t}-v_{t}}{v_{t}}, \quad t=0, \ldots, n .
$$

The sequence $v_{0}, \ldots, v_{n}$ thus satisfies the recursion

$$
v_{0}=\left(1+e_{0}\right) F_{0}, \quad v_{t}=\left(1+e_{t}\right) F_{t}\left(v_{0}, \ldots, v_{t-1}\right), \quad t=1, \ldots, n .
$$

In typical applications the errors $e_{t}, e_{t}^{\prime}$ are bounded in modulus by the floatingpoint accuracy constant $\eta$ or some multiple of $\eta$. We shall call the $e_{t}$ local (rounding) errors.

For the sake of notational simplicity, let us introduce the notation $J_{w}$ for the diagonal matrix $\operatorname{diag}\left(w_{0}, \ldots, w_{n}\right)$ and any vector $w=\left(w_{0}, \ldots, w_{n}\right)$. In this sense,

$$
J_{w}^{-1}=\operatorname{diag}\left(\frac{1}{w_{0}}, \ldots, \frac{1}{w_{n}}\right),
$$

provided that $w_{t} \neq 0, t=0, \ldots, n$. Using this notation, the absolute and relative a priori and a posteriori errors of approximations $v=\left(v_{0}, \ldots, v_{n}\right)$ of $u=$ $\left(u_{0}, \ldots, u_{n}\right)$, where $u_{t} \neq 0, v_{t} \neq 0$ for all $t$, satisfy the relations

$$
\begin{array}{lll}
\Delta u=v-u, & P u=J_{u}^{-1} \Delta u, & \Delta u=J_{u} P u, \\
\Delta v=u-v, & P v=J_{v}^{-1} \Delta v, & \Delta v=J_{v} P v .
\end{array}
$$

Now the fundamental error equations for the solutions of the perturbed algorithm shall be established. The functions $F_{t} \in(F)$ are Fréchet-differentiable at each interior point of their domains of definition and satisfy the Taylor formula

$$
F_{t}(x+h)=F_{t}(x)+F_{t}^{\prime}(x) h+R_{t}(x, h) .
$$


The gradients $F_{t}^{\prime}(x)$ of $F_{t}$ have the form

$$
F_{t}^{\prime}(x)=\left(\frac{\partial F_{t}}{\partial x_{0}}(x), \ldots, \frac{\partial F_{t}}{\partial x_{t-1}}(x), 0, \ldots, 0\right),
$$

because $F_{t}$ depends on $x_{0}, \ldots, x_{t-1}$ only. Input operations $F_{t} \in(\mathrm{F} 0)$ are constant functions so that

$$
F_{t}^{\prime}(x)=0, \quad R_{t}(x ; h)=0 .
$$

For elementary functions $F_{t}(x)=f\left(x_{j}\right)$ one obtains, from 1.1.(10) with $a=x_{j}, a^{\prime}=$ $x_{j}^{\prime}=x_{j}+h_{j}$, the representation

$$
F_{t}^{\prime}(x) h=f^{\prime}\left(x_{j}\right) h_{j}, \quad R_{t}(x ; h)=f\left[x_{j}, x_{j}, x_{j}^{\prime}\right] h_{j}^{2},
$$

where it is assumed that the line segment $\overline{x_{j} x_{j}^{\prime}}$ belongs to def $f$. Additions and subtractions $F_{t}(x)=x_{i} \pm x_{j}$ give

$$
F_{t}^{\prime}(x) h=h_{i} \pm h_{j}, \quad R_{t}(x ; h)=0 .
$$

For multiplications $F_{t}(x)=x_{i} x_{j}$ it is seen from 1.1(3) that

$$
F_{t}^{\prime}(x) h=x_{j} h_{i}+x_{i} h_{j}, \quad R_{t}(x ; h)=h_{i} h_{j} \text {. }
$$

In the case of divisions one finally has

$$
F_{t}^{\prime}(x) h=\frac{1}{x_{j}} h_{i}-\frac{x_{i}}{x_{j}^{2}} h_{j}, \quad R_{t}(x ; h)=\frac{h_{j}}{x_{j}^{\prime}} F_{t}^{\prime}(x) h,
$$

provided that $x_{j} \neq 0, x_{j}^{\prime} \neq 0$.

The next theorem constitutes the basis for subsequent error estimates. It shows that the remainder term of the Taylor formula can be estimated locally uniformly in suitable neighborhoods of $u$.

(12) Let the solution $u$ of the algorithm (A) be an interior point of def $F_{t}$ and $u_{t} \neq 0$ for all $t$. Then there exist positive constants $\kappa_{t}, \xi_{t}$ such that uniformly for all $x, h$ in

$$
\left|\frac{x_{t}-u_{t}}{u_{t}}\right| \leqslant \xi_{t}, \quad\left|\frac{h_{t}}{u_{t}}\right| \leqslant \xi_{t}, \quad t=0, \ldots, n,
$$

the joins $\overline{x, x+h}$ belong to $\operatorname{def} F_{t}^{\prime}$, the components $x_{t}$ do not vanish, and the remainder terms satisfy the estimates

$$
\left|\frac{1}{F_{t}(x)} R_{t}(x ; h)\right| \leqslant \kappa_{t}\left(\max _{j<t}\left|P x_{j}\right|\right)^{2}, \quad t=0, \ldots, n,
$$

where $P x_{j}=h_{j} / x_{j}$.

Proof. (i) As $u$ is an interior point of def $F_{t}$ for all $t$, there are positive constants $\xi_{t}^{0} \leqslant 2 / 3$ such that all $x$ in the neighborhood $\left|x_{t}-u_{t}\right| /\left|u_{t}\right|<\xi_{t}^{0}, t=0, \ldots, n$, of $u$ belong to def $F_{t}^{\prime}$ for all $t$. Consider first those indices $t$ such that $F_{t} \in(\mathrm{F} 1)$, that is, $F_{t}(x)=f_{t}\left(x_{j_{t}}\right)$. Then $u_{j_{t}}$ is an interior point of def $f_{t}$ and there are positive constants $\xi_{j_{t}}^{1} \leqslant \xi_{j_{i}}^{0}$ such that all $x_{j_{t}}$ satisfying $\left|x_{j_{t}}-u_{j_{t}}\right| /\left|u_{j_{t}}\right|<\xi_{j_{t}}^{1}$ belong to def $f_{t}$, where $f_{t}$ is twice continuously differentiable. Since $f_{t}\left(u_{j_{i}}\right)=u_{t} \neq 0, \xi_{j}^{1}$ may be chosen so small that also $f_{t}\left(x_{j_{i}}\right) \neq 0$ for $x_{j_{t}}$ in this neighborhood. For all indices $k \neq j_{t}$ and $F_{t} \in(\mathrm{F} 1)$ put $\xi_{k}^{1}=\xi_{k}^{0}$. Finally let $\xi_{t}=\frac{1}{2} \xi_{t}{ }^{1}$ for $t=0, \ldots, n$.

(ii) By virtue of the condition $\xi_{t} \leqslant \frac{1}{2} \xi_{t}^{0}, t=0, \ldots, n$, the line segments $\overline{x, x+h}$ belong to def $F_{t}^{\prime}$ for all $t=0, \ldots, n$, whenever $x, h$ is in the neighborhood (12i) of 
$u, 0$. Since $\xi_{t}<1, x_{t} \neq 0$ for all $t$. For input operations, additions and subtractions, (12ii) holds trivially with $\kappa_{t}=0$. In view of (10), the remainder term estimate then holds for multiplications with $\kappa_{t}=1$. In the case of divisions one has

$$
\frac{1}{F_{t}(x)} R_{t}(x ; h)=-\frac{1}{1+P x_{j}}\left(P x_{i}-P x_{j}\right) P x_{j}, \quad i=i_{t}, j=j_{t} .
$$

Now $\xi_{k} \leqslant \frac{1}{2} \xi_{k}^{0} \leqslant \frac{1}{3}$ for all $k$, and consequently

$$
\frac{2}{3} \leqslant 1-\left|\frac{x_{k}-u_{k}}{u_{k}}\right| \leqslant\left|\frac{x_{k}}{u_{k}}\right|, \quad\left|P x_{k}\right|=\left|\frac{h_{k}}{u_{k}}\right|\left|\frac{u_{k}}{x_{k}}\right|<\frac{1}{2} .
$$

Hence the estimate (12ii) is true for $\kappa_{t}=4$. Finally, consider those $t$ such that $F_{t} \in(\mathrm{F} 1)$, that is, $F_{t}(x)=f_{t}\left(x_{j_{t}}\right)$. The estimate (12ii) then follows from (8) and 1.1(11) using the constants

$$
\kappa_{t}=\frac{1}{2} \max \frac{x_{j}^{2}}{\left|f_{t}\left(x_{j}\right)\right|} \max \left|f_{t}^{\prime \prime}\left(z_{j}\right)\right|
$$

the maxima being taken over all $x_{j}, z_{j}$ such that

$$
\left|\frac{x_{j}-u_{j}}{u_{j}}\right| \leqslant \xi_{j}, \quad\left|\frac{z_{j}-u_{j}}{u_{j}}\right| \leqslant 2 \xi_{j}, \quad j=j_{t} .
$$

Applying Taylor's formula to the solutions $x=u, x+h=v$ of the algorithm (A) and its perturbation $(\tilde{\mathrm{A}})$ gives

$$
F_{t}(v)=F_{t}(u)+F_{t}^{\prime}(u) \Delta u+R_{t}(u ; \Delta u),
$$

where $h=v-u=\Delta u$, and thus

$$
v_{t}=\left(1+e_{t}\right)\left\{u_{t}+F_{t}^{\prime}(u) \Delta u+R_{t}(u ; \Delta u)\right\}
$$

The absolute a priori errors $\Delta u_{t}=v_{t}-u_{t}$ therefore satisfy the system of equations

$$
\Delta u_{0}=u_{0} e_{0}, \quad \Delta u_{t}-F_{t}^{\prime}(u) \Delta u=u_{t} e_{t}+T_{t}, \quad t=1, \ldots, n,
$$

and the relative a priori errors $P u_{t}=\left(v_{t}-u_{t}\right) / u_{t}$ the system

$$
P u_{0}=e_{0}, \quad P u_{t}-\frac{1}{u_{t}} F_{t}^{\prime}(u) J_{u} P u=e_{t}+\frac{1}{u_{t}} T_{t}, \quad t=1, \ldots, n,
$$

using the remainder terms

$$
T_{t}=e_{t} F_{t}^{\prime}(u) \Delta u+\left(1+e_{t}\right) R_{\imath}(u ; \Delta u) .
$$

Similarly, the Taylor formula can be applied for $x=v, x+h=u$ and $h=u-$ $v=\Delta v$. Then

$$
F_{t}(u)=F_{t}(v)+F_{t}^{\prime}(v) \Delta v+R_{t}(v ; \Delta v) .
$$

From $(\mathrm{A}),(\tilde{\mathrm{A}})$, and $(2)$ it is seen that $F_{t}(u)=u_{t}$ and

$$
F_{t}(v)=\left(1+e_{t}^{\prime}\right) v_{t}, \quad \frac{1}{v_{t}}=\frac{1}{F_{t}(v)}-\frac{e_{t}}{v_{t}}, \quad e_{t}^{\prime}=-\frac{e_{t}}{1+e_{t}} .
$$

Hence the absolute a posteriori errors $\Delta v_{t}=u_{t}-v_{t}$ satisfy the equations

$$
\Delta v_{0}=v_{0} e_{0}^{\prime}, \quad \Delta v_{t}-F_{t}^{\prime}(v) \Delta v=v_{t} e_{t}^{\prime}+R_{t}(v ; \Delta v), \quad t=1, \ldots, n .
$$

By (2), $e_{t}^{\prime}$ denotes the relative a posteriori error of the approximation $v_{t}$ of $F_{t}(v)$. Dividing both sides of (17) by $v_{t}$ yields the equations

$$
P v_{0}=e_{0}^{\prime}, \quad P v_{t}-\frac{1}{v_{t}} F_{t}^{\prime}(v) J_{v} P v=e_{t}^{\prime}+\frac{1}{v_{t}} R_{t}(v ; \Delta v), \quad t=1, \ldots, n,
$$


for the relative a posteriori errors $P v_{t}=\left(u_{t}-v_{t}\right) / v_{t}$. Approximating $v_{t}$ by $F_{t}(v)$ yields the corresponding systems

$$
\begin{aligned}
& \Delta v_{0}=v_{0} e_{0}^{\prime}, \quad \Delta v_{t}-\frac{v_{t}}{F_{t}(v)} F_{t}^{\prime}(v) \Delta v=v_{t} e_{t}^{\prime}+R_{t}^{\prime}, \\
& P v_{0}=e_{0}^{\prime}, \quad P v_{t}-\frac{1}{F_{t}(v)} F_{t}^{\prime}(v) J_{v} P v=e_{t}^{\prime}+\frac{1}{v_{t}} R_{t}^{\prime},
\end{aligned}
$$

for $t=1, \ldots, n$, where $R_{t}^{\prime}$ denotes the remainder terms

$$
R_{t}^{\prime}=-e_{t} F_{t}^{\prime}(v) \Delta v+R_{t}(v ; \Delta v) \text {. }
$$

In (17), (18), (19), additionally, $e_{t}^{\prime}$ can be approximated by $-e_{t}$ and the associated remainder $-e_{t} e_{t}^{\prime}$ be added to $R_{t}^{\prime}$.

The above error equations have the general form

$$
z_{0}=f_{0}, \quad z_{t}-\sum_{k=0}^{t-1} b_{t k} z_{k}=f_{t}, \quad t=1, \ldots, n .
$$

The coefficients $b_{t k}$ vanish for $k>t$ because $\partial F_{t} / \partial x_{k}=0$ due to (6). The coefficients $b_{t k}^{\text {abs }}, b_{t k}^{\text {rel }}$ of the equations (14), (15), for $z_{t}=\Delta u_{t}, P u_{t}$ read

$$
b_{t k}^{\mathrm{abs}}=\frac{\partial F_{t}}{\partial x_{t}}(u), \quad b_{t k}^{\mathrm{rel}}=\frac{u_{k}}{u_{t}} \frac{\partial F_{t}}{\partial x_{k}}(u), \quad t, k=0, \ldots, n .
$$

For input operations, by (7),

$$
b_{t k}^{\mathrm{abs}}=b_{t k}^{\mathrm{rel}}=0
$$

and for 'built-in' functions

$$
b_{t k}^{\mathrm{abs}}=f^{\prime}\left(x_{j}\right) \delta_{j k}, \quad b_{t k}^{\mathrm{rel}}=\frac{x_{j}}{f\left(x_{j}\right)} f^{\prime}\left(x_{j}\right) \delta_{j k}, \quad j=j_{t} .
$$

The coefficients $b_{t k}^{\text {abs }}$ of the arithmetic operations $F_{t} \in(\mathrm{F} 2)$ are listed in Table 1.2. Note that the coefficients for additions and subtractions are particularly simple in

\begin{tabular}{|c|c|c|c|c|c|}
\hline 0 & $k=i$ & $k=j$ & $k=i$ & $k=j$ & $k \neq i, j$ \\
\hline+ & 1 & 1 & $\frac{u_{i}}{u_{t}}$ & $\frac{u_{j}}{u_{t}}$ & 0 \\
\hline- & 1 & -1 & $\frac{u_{i}}{u_{t}}$ & $-\frac{u_{j}}{u_{t}}$ & 0 \\
\hline$x$ & $u_{j}$ & $u_{i}$ & 1 & 1 & 0 \\
\hline / & $\frac{1}{u_{j}}$ & $-\frac{u_{t}}{u_{j}}$ & 1 & -1 & 0 \\
\hline
\end{tabular}
the absolute a priori error equations, and for multiplications and divisions in the relative a priori error equations. This fact can be used advantageously in the error analysis of specific examples.

\section{TABLE 1.2}

Matrix elements in the absolute and relative a priori error equations where $i=i_{t}, j=j_{t}$ 
The representation of the matrix elements in Table 1.2 is so chosen that it also yields easily computable coefficients of a posteriori error equations. These are obtained by replacing $u_{i}, u_{j}, u_{t}$ by the solutions $v_{i}, v_{j}, v_{t}$ of the perturbed algorithm $(\tilde{\mathrm{A}})$. In this way, the absolute a posteriori error equations for additions, subtractions, and multiplications are given the coefficients

$$
b_{t k}^{\mathrm{abs}}=\frac{\partial F_{t}}{\partial x_{k}}(v), \quad k=0, \ldots, n,
$$

and for divisions $F_{t}(v)=v_{i} / v_{j}$,

$$
b_{t k}^{\mathrm{abs}}=\frac{\partial F_{t}}{\partial x_{k}}(v), \quad k \neq j ; \quad b_{t j}^{\mathrm{abs}}=\frac{v_{t}}{F_{t}(v)} \frac{\partial F_{t}}{\partial x_{j}}(v) .
$$

The relative a posteriori error equations for additions and subtractions take on the form (18), that is, (21) has the coefficients

$$
b_{t k}^{\mathrm{rel}}=\frac{v_{k}}{v_{t}} \frac{\partial F_{t}}{\partial x_{k}}(v), \quad k=0, \ldots, n,
$$

whereas for multiplications and divisions the form (19) is obtained such that the coefficients in (21) become

$$
b_{t k}^{\mathrm{rel}}=\frac{v_{k}}{F_{t}(v)} \frac{\partial F_{t}}{\partial x_{k}}(v), \quad k=0, \ldots, n .
$$

An error estimate for the solutions of these modified a posteriori error equations will be given in Theorem 2.2(15).

1.3. The Linear Error Equations. Fundamental notions of the perturbation theory for evaluation algorithms are the associated mappings $F$ and $A=I-F$ in $\mathbf{R}^{n+1}$ that will be introduced now. The functions $F_{0}, \ldots, F_{n}$, specifying the algorithm (A), constitute the vector-valued mapping

$$
F(x)=\left(F_{0}(x), \ldots, F_{n}(x)\right)
$$

for all $x \in \mathbf{R}^{n+1}$ such that $x \in \operatorname{def} F_{t}$ for all $t$. The solution $u=\left(u_{0}, \ldots, u_{n}\right)$ of the algorithm (A) may thus be viewed as fixed point of $F$,

$$
u=F(u) \text {. }
$$

Let $v=\left(v_{0}, \ldots, v_{n}\right)$ denote the solution of the perturbed algorithm ( $\left.\tilde{\mathrm{A}}\right)$. By inserting $v$ into (2), the associated residual $d=v-F(v)$ is determined. In view of 1.2.( $\tilde{\mathrm{A}})$, obviously,

$$
d_{t}=e_{t} F_{t}(v)=-e_{t}^{\prime} v_{t}, \quad t=0, \ldots, n
$$

that is, $d=-J_{v} e^{\prime}$.

The algorithm (A) defines uniquely the mapping

$$
A x=x-F(x)=\left(A_{0} x, \ldots, A_{n} x\right),
$$

having the components

$$
A_{0} x=x_{0}-F_{0}, \quad A_{t} x=x_{t}-F_{t}(x), \quad t=1, \ldots, n,
$$

for $x \in \operatorname{def} A=\operatorname{def} F$. The solution $u$ of the unperturbed algorithm (A) is, by (2), a solution of the functional equation

$$
A u=0,
$$


and the solution $v$ of the perturbed algorithm $(\tilde{\mathrm{A}})$ satisfies the equation

$$
A v=d,
$$

using the residual vector (3). In this way, the error analysis of the perturbed algorithm becomes a perturbation theory of the mapping $A$ in a neighborhood of $u$.

From Section 1.2 it follows that the mappings $F, A$ are Fréchet-differentiable at each interior point of their domain of definition. The derivative $F^{\prime}(x)$ of $F$ is represented by the matrix

$$
F^{\prime}(x)=\left(\frac{\partial F_{t}}{\partial x_{k}}(x)\right)_{t, k=0, \ldots, n},
$$

and the associated remainder term by

$$
R^{F}(x ; h)=F(x+h)-F(x)-F^{\prime}(x) h=\left(R_{t}(x ; h)\right)_{t=0, \ldots, n},
$$

where $R_{t}$ denotes the remainder terms of the Taylor formula 1.2(5). Hereby also the mapping $A$ is differentiable at all interior points of $\operatorname{def} A=\operatorname{def} F$, where

$$
A^{\prime} x=I-F^{\prime}(x), \quad x \in \operatorname{def} F^{\prime},
$$

and

$$
R^{A}(x ; h)=A(x+h)-A x-\left(A^{\prime} x\right) h=-R^{F}(x ; h) .
$$

The derivative $A^{\prime} u$ is called the derivative of the algorithm (A) and correspondingly $A^{\prime} v$ the derivative of the perturbed algorithm $(\tilde{\mathrm{A}})$. From the representation 1.2(6) of the gradients $F_{t}^{\prime}(x)$ it is seen that the matrix of $A^{\prime} x$ is lower triangular and its diagonal elements are equal to 1 . Consequently,

(11) $A^{\prime} x$ is a bijective linear mapping of $\mathbf{R}^{n+1}$ for each $x \in \operatorname{def} A^{\prime}=\operatorname{def} F^{\prime}$.

Using these mappings, the absolute and relative a priori error equations 1.2(14), (15) may be written in the concise form

$$
\left(A^{\prime} u\right) \Delta u=J_{u} e+T, \quad\left(A^{\prime} u\right)_{\text {rel }} P u=e+J_{u}^{-1} T .
$$

Analogously, the absolute and relative a posteriori error equations 1.2(17), (18) read

$$
\left(A^{\prime} v\right) \Delta v=J_{v} e^{\prime}+R, \quad\left(A^{\prime} v\right)_{\mathrm{rel}} P v=e^{\prime}+J_{v}^{-1} R,
$$

where

$$
\left(A^{\prime} u\right)_{\mathrm{rel}}=J_{u}^{-1}\left(A^{\prime} u\right) J_{u}, \quad\left(A^{\prime} v\right)_{\mathrm{rel}}=J_{v}^{-1}\left(A^{\prime} v\right) J_{v} .
$$

We shall see in Section 2.1 that the remainder terms $R, T$ are of second order in $\varepsilon$ as long as the local errors are bounded by $\left|e_{t}\right|<\varepsilon,\left|e_{t}^{\prime}\right|<\varepsilon$, and $\varepsilon$ is sufficiently small. Hence neglecting the remainder term $T$ in (12) yields the associated linear $a$ priori error equations

$$
\left(A^{\prime} u\right) s=J_{u} e, \quad\left(A^{\prime} u\right)_{\mathrm{rel}} r=e .
$$

Similarly, by neglecting $R$ in (13), the linear a posteriori error equations

$$
\left(A^{\prime} v\right) s=J_{v} e^{\prime}, \quad\left(A^{\prime} v\right)_{\mathrm{rel}} r=e^{\prime}
$$

are found. We shall prove in Chapter 2 that the solutions $s, r$ of (15) approximate the a priori errors $\Delta u, P u$ and the solutions $s, r$ of (16) approximate the a posteriori errors $\Delta v, P v$. 
The eight linear systems (12), (13), (15), (16) have, by (9), (14), the general form

$$
z-B z=f
$$

using the mappings

\begin{tabular}{c|c|c}
$B$ & absolute & relative \\
\hline a priori & $F^{\prime}(u)$ & $J_{u}^{-1} F^{\prime}(u) J_{u}$ \\
\hline a posteriori & $F^{\prime}(v)$ & $J_{v}^{-1} F^{\prime}(v) J_{v}$
\end{tabular}

Explicit expressions for elements $b_{t k}$ of the associated matrices of these mappings $B$ have been listed already in Table 1.2. The solutions $z$ in (12), (13) are given by

\begin{tabular}{c|c|c}
$z$ & absolute & relative \\
\hline a priori & $\Delta u$ & $P u$ \\
\hline a posteriori & $\Delta v$ & $P v$
\end{tabular}

The right-hand sides of the linear error equations (15), (16) read

\begin{tabular}{c|c|c}
$f$ & absolute & relative \\
\hline a priori & $J_{u} e$ & $e$ \\
\hline a posteriori & $J_{v} e^{\prime}$ & $e^{\prime}$
\end{tabular}

As we have stated above, $A^{\prime} u, A^{\prime} v$ and thus $\left(A^{\prime} u\right)_{\text {rel }},\left(A^{\prime} v\right)_{\text {rel }}$ are bijective linear mappings. In order to simplify notation in the further study, the associated inverse operators are called solution operators and are denoted by $L$. The solutions of the above eight linear systems are then written

$$
z=L f, \quad L=(I-B)^{-1} .
$$

In the case of the a priori error equations,

$$
L^{\mathrm{abs}}=\left(A^{\prime} u\right)^{-1}, \quad L^{\mathrm{rel}}=\left(A^{\prime} u\right)_{\mathrm{rel}}^{-1},
$$

and, consequently,

$$
L^{\mathrm{abs}}=J_{u} L^{\mathrm{rel}} J_{u}^{-1}, \quad L^{\mathrm{rel}}=J_{u}^{-1} L^{\mathrm{abs}} J_{u} .
$$

Correspondingly, for the associated a posteriori error equations

$$
L^{\mathrm{abs}}=\left(A^{\prime} v\right)^{-1}, \quad L^{\mathrm{rel}}=\left(A^{\prime} v\right)_{\mathrm{rel},}^{-1}
$$

whence

$$
L^{\mathrm{abs}}=J_{v} L^{\mathrm{rel}} J_{v}^{-1}, \quad L^{\mathrm{rel}}=J_{v}^{-1} L^{\mathrm{abs}} J_{v} .
$$

The representation $L=(I-B)^{-1}$ immediately yields the relations

$$
L=B L+I=L B+I \text {. }
$$

Componentwise, the first relation reads

$$
L_{t k}=\sum_{l=k}^{t-1} b_{t l} L_{l k}+\delta_{t k}, \quad k=0, \ldots, t,
$$

because

$$
b_{i k}=0, \quad i<k, \quad L_{i k}=0, \quad i<k .
$$

A recurrence of this type for computing the entries of the matrix $L$ is found in Henrici [8, (16-19)]. From the second relation in (26) one obtains

$$
L_{t t}=1, \quad L_{t k}=\sum_{l=k+1}^{t} L_{t l} b_{l k}, \quad k=0, \ldots, t-1 .
$$


Using the row vectors

$$
L_{t}=\left(L_{t k}\right)_{k=0, \ldots, n}, \quad b_{t}=\left(b_{t k}\right)_{k=0, \ldots, n}, \quad \delta_{t}=\left(\delta_{t 0}, \ldots, \delta_{t n}\right),
$$

we can write $L=L B+I$ in the form

$$
L_{t}=\sum_{l=1}^{t} L_{t l} b_{l}+\delta_{t}
$$

Now, put

$$
L_{t}^{(j)}=\sum_{l=j+1}^{t} L_{t l} b_{l}+\delta_{t}, \quad j=0, \ldots, t .
$$

From (28), (29) it is seen that

$$
L_{t k}^{(j)}=\sum_{l=k+1}^{t} L_{t l} b_{l k}=L_{t k}, \quad j \leqslant k, k=0, \ldots, t-1,
$$

thus, in particular, $L_{t j}=L_{t j}^{(j)}$. Consequently, the row $L_{t}$ of the matrix $L$ can be computed recurrently from

$$
L_{t}^{(t)}=\delta_{t}, \quad L_{t}^{(j-1)}=L_{t}^{(j)}+L_{t_{j}}^{(j)} b_{j}, \quad j=t, \ldots, 1,
$$

and

$$
L_{t}=L_{t}^{(0)}
$$

This is the algorithm (T) of Linnainmaa [10] applied to the computation of the row $L_{t}$ of the matrix $L$ from the rows of the matrix $B$. The same algorithm has been proposed by Larson-Sameh [9] in a somewhat different setting.

2. Condition Numbers, Error Estimates, Graphs. In Sections 2.1, 2.2 it will be shown that the solutions $s, r$ of the linear a priori and a posteriori error equations yield approximations of the a priori errors $\Delta u, P u$ and a posteriori errors $\Delta v, P v$. Simultaneously, estimates of these errors will be obtained. A fundamental tool of the error analysis is the notion of condition number. The error approximations $s_{t}, r_{t}$ are linear forms in the local errors specifying the perturbations of the algorithm. Our condition numbers are norms of these linear forms with respect to suitably chosen weighted maximum norms over the space of local errors. Therefore condition numbers are optimal bounds of the error approximations $s_{t}, r_{t}$ for all local errors in the considered distribution. Consequently, also the associated estimates of the errors $\Delta u, P u$ and $\Delta v, P v$ are optimal if terms $O_{t}(\varepsilon)$ are neglected against 1 . In addition, Section 2.2 demonstrates that the solutions of the linear a posteriori error equations are approximations of the associated solutions of the a priori error equations and thus the a posteriori condition numbers approximations of the a priori condition numbers. Moreover, it will be shown that, using solutions of the linear a posteriori error equations, approximations of about double precision can be computed.

Each algorithm and its uniquely associated system of linear error equations determines a graph, defined in Section 2.3. Graphs constitute useful means of the error analysis, particularly also for deriving condition numbers of the algorithm. For example, if the graph is a tree, the condition numbers can be determined a priori and be computed a posteriori by simple recursion formulae. In all cases, bounds for condition numbers and hence for error estimates can recursively be obtained and computed a posteriori. For examples we refer to Stummel [17]-[23]. 
2.1. A Priori Condition Numbers and Error Estimates. In the preceding sections the general linear error equations have been established for the class of algorithms considered here. Using the associated solution operators $L=(I-B)^{-1}$, the solutions $z$ of these linear systems have the form $z=L f$. Due to $1.2(6), 1.3(18)$, the mappings $B$ are represented by lower-triangular matrices with zeros as diagonal elements. Consequently, the matrices of the solution operators are lower triangular with all diagonal elements equal to 1 . By $L_{i k}$ are meant the $(n+1)^{2}$ elements of the matrix of $L$. Then

$$
z_{t}=f_{t}+\sum_{k=0}^{t-1} L_{t k} f_{k}, \quad t=0, \ldots, n,
$$

as

$$
L_{t t}=1, \quad L_{t k}=0, \quad t<k .
$$

For each $t$ the right-hand side of (1) is a linear form $L_{t}$ over $\mathbf{R}^{n+1}$. Evidently,

$$
L_{t}=\operatorname{pr}_{t} L \text {, }
$$

where $\mathrm{pr}_{t}$ is the projection onto the $t$ th coordinate axis. The solutions $s_{t}, r_{t}$ of the linear a priori error equations 1.3(15) then have the representation

$$
s_{t}=L_{t}^{\mathrm{abs}} J_{u} e, \quad r_{t}=L_{t}^{\mathrm{rel}} e .
$$

Of fundamental importance in the following are the associated absolute and relative a priori condition numbers

$$
\sigma_{t}^{1}=\sum_{k=0}^{t}\left|L_{t k}^{\mathrm{abs}} u_{k}\right|, \quad \rho_{t}^{1}=\sum_{k=0}^{t}\left|L_{t k}^{\mathrm{rel}}\right|, \quad t=0, \ldots, n .
$$

From $L_{t t}=1$ it follows that

$$
\rho_{0}^{1}=1, \quad \rho_{t}^{1} \geqslant 1 \text {. }
$$

By virtue of 1.3(23),

$$
L^{\mathrm{abs}} J_{u}=J_{u} L^{\mathrm{rel}}
$$

thus

$$
s_{t}=u_{t} r_{t}, \quad \sigma_{t}^{1}=\left|u_{t}\right| \rho_{t}^{1} .
$$

Using the above condition numbers, the solutions $s_{t}, r_{t}$ satisfy the inequalities

$$
\left|s_{t}\right| \leqslant \sigma_{t}^{1} \varepsilon, \quad\left|r_{t}\right| \leqslant \rho_{t}^{1} \varepsilon,
$$

for all $\left|e_{k}\right| \leqslant \varepsilon, k=0, \ldots, n$. These estimates are sharp or optimal because the bounds $\sigma_{t}^{1} \varepsilon, \rho_{t}^{1} \varepsilon$ are attained for the local error distributions

$$
e_{k}^{\mathrm{abs}}=\varepsilon \operatorname{sgn}\left(L_{t k}^{\mathrm{abs}} u_{k}\right), \quad e_{k}^{\mathrm{rel}}=\varepsilon \operatorname{sgn}\left(L_{t k}^{\mathrm{rel}}\right), \quad k=0, \ldots, n .
$$

By these means we are now in the position to establish error estimates for the approximations $s, r$ of the a priori errors $\Delta u, P u$. By 1.3(12), (15), (22), obviously,

$$
\Delta u_{t}-s_{t}=L_{t}^{\mathrm{abs}} T, \quad P u_{t}-r_{t}=L_{t}^{\mathrm{rel}} J_{u}^{-1} T,
$$

whence, using (4),

$$
\left|\Delta u_{t}-s_{t}\right|<\sigma_{t}^{1} \max _{j<t}\left|\frac{1}{u_{j}} T_{j}\right|, \quad\left|P u_{t}-r_{t}\right|<\rho_{t}^{1} \max _{j<t}\left|\frac{1}{u_{j}} T_{j}\right| .
$$


We assume throughout the paper that the solution $u$ of $(\mathrm{A})$ is an interior point of def $F_{t}$ and $u_{t} \neq 0$ for all $t$. The first main theorem of the paper then reads:

(12) For all local errors $\left|e_{t}\right| \leqslant \varepsilon, t=0, \ldots, n$, and sufficiently small $\varepsilon$ the perturbed algorithm $(\tilde{\mathrm{A}})$ is well defined. The solutions $s, r$ of the linear a priori error equations are approximations of the absolute and relative errors $\Delta u, P u$ with the associated error estimates

$$
\left|\Delta u_{t}-s_{t}\right| \leqslant \sigma_{t}^{1} \omega_{t} \varepsilon^{2}, \quad\left|P u_{t}-r_{t}\right| \leqslant \rho_{t}^{1} \omega_{t} \varepsilon^{2}, \quad t=0, \ldots, n .
$$

In particular, $\Delta u, P u$ permit the estimates

$$
\left|\Delta u_{t}\right| \leqslant\left(1+\omega_{t} \varepsilon\right) \sigma_{t}^{1} \varepsilon, \quad\left|P u_{t}\right| \leqslant\left(1+\omega_{t} \varepsilon\right) \rho_{t}^{1} \varepsilon, \quad t=0, \ldots, n .
$$

Proof. (i) First let us define the constants

$$
\beta_{t}^{\prime}=\max _{j<t} \sum_{k=0}^{j-1}\left|b_{j k}^{\mathrm{rel}}\right|, \quad \kappa_{t}^{\prime}=\max _{j<t} \kappa_{j}
$$

where $\kappa_{j}$ denotes the constants in the remainder estimates $1.2(12)$ at the point $u$. Next let $\beta=\beta_{n}^{\prime}, \kappa=\kappa_{n}^{\prime}$, and $\rho=\max \rho_{t}^{1}$. For arbitrary but fixed $\vartheta>0$ put

$$
\omega=(\beta+(1+\vartheta) \kappa \tau) \tau, \quad \tau=(1+\vartheta) \rho .
$$

As $u$ is an interior point of $\operatorname{def} F$, there exists a positive constant $\xi$ such that for each $t$ the statement

$$
\left|x_{j}-u_{j}\right| \leqslant \xi\left|u_{j}\right|, \quad j=0, \ldots t-1 \Rightarrow\left(x_{0}, \ldots, x_{t-1}\right) \in \operatorname{def} F_{t}
$$

is true and the estimates 1.2(12) are applicable for $x=u$ and all $\left|h_{j}\right| \leqslant \xi\left|u_{j}\right|$, $j=0, \ldots, n$. Finally, let

$$
\varepsilon \leqslant \varepsilon_{0}=\min \left(\vartheta, \frac{\vartheta}{\omega}, \frac{\xi}{\tau}\right)
$$

(ii) The theorem will be proved by finite induction. For $t=0, P u_{0}=r_{0}=e_{0}$ and $\rho_{0}^{1}=1$, so that the inequalities (12i), (12ii) are valid with $\omega_{0}=0$. For $t>1$ assume now that the proposition

$$
\left|v_{j}-u_{j}\right| \leqslant \xi\left|u_{j}\right|, \quad\left|P u_{j}\right| \leqslant\left(1+\omega_{j} \varepsilon\right) \rho_{j}^{1} \varepsilon, \quad \omega_{j}<\omega,
$$

is true for $j=0, \ldots, t-1$. From (8), (11) it then follows that

$$
\left|P u_{t}\right| \leqslant\left(1+\frac{1}{\varepsilon} \max _{j<t}\left|\frac{1}{u_{j}} T_{j}\right|\right) \rho_{t}^{1} \varepsilon,
$$

using the remainder terms $T_{j}$ in $1.2(16)$, that is,

$$
\frac{1}{u_{j}} T_{j}=e_{j} B_{j}^{\mathrm{rel}} P u+\left(1+e_{j}\right) \frac{1}{u_{j}} R_{j}\left(u_{j} ; \Delta u\right) .
$$

On setting

$$
\omega_{t-1}^{\prime}=\max _{j<t} \omega_{j}, \quad \rho_{t-1}^{\prime}=\max _{j<t} \rho_{j}^{1}, \quad \tau_{t}=\left(1+\omega_{t-1}^{\prime} \varepsilon\right) \rho_{t-1}^{\prime},
$$

the above proposition and Theorem 1.2(12) imply

$$
\max _{j<t}\left|P u_{j}\right| \leqslant \tau_{t} \varepsilon, \quad \max _{j<t}\left|\frac{1}{u_{j}} T_{j}\right| \leqslant \tau_{t}\left(\beta_{t}^{\prime}+(1+\varepsilon) \kappa_{t}^{\prime} \tau_{t}\right) \varepsilon^{2} .
$$


Consequently,

$$
\max _{j<t}\left|\frac{1}{u_{j}} T_{j}\right| \leqslant \omega_{t} \varepsilon^{2}
$$

where $\omega_{t}=\tau_{t}\left(\beta_{t}^{\prime}+(1+\varepsilon) \kappa_{t}^{\prime} \tau_{t}\right), t=1, \ldots, n$. Finally, the above proposition entails $\omega_{t-1}^{\prime} \leqslant \omega, \tau_{t} \leqslant(1+\omega \varepsilon) \rho \leqslant \tau$. Thus $\omega_{t} \leqslant(\beta+(1+\vartheta) \kappa \tau) \tau=\omega$ and

$$
\left|\frac{v_{t}-u_{t}}{u_{t}}\right|=\left|P u_{t}\right| \leqslant\left(1+\omega_{t} \varepsilon\right) \rho_{t}^{1} \varepsilon \leqslant \tau \varepsilon \leqslant \xi
$$

for all $\varepsilon \leqslant \varepsilon_{0}$. Hence the above proposition is true also for $j=t$ and consequently for all $j=0, \ldots, n$. The estimates of the remainder terms $T_{t} / u_{t}$, proved above, immediately yield the error estimates (12i), (12ii).

The above theorem guarantees that the solutions $s, r$ of the linear error equations are equal to the a priori errors $\Delta u, P u$ save for terms of second order in $\varepsilon$,

$$
\Delta u_{t}=s_{t}+O_{t}\left(\varepsilon^{2}\right), \quad P u_{t}=r_{t}+O_{t}\left(\varepsilon^{2}\right)
$$

On this basis, error estimates can be derived regarding specific distributions of the local errors. Let us assume that

$$
\left|e_{t}\right| \leqslant \gamma_{t} \eta, \quad t=0, \ldots, n,
$$

where $\gamma_{0}, \ldots, \gamma_{n}$ are appropriate nonnegative weights. Theorem (12) applies to this specific distribution of local errors in choosing $\varepsilon=\max \gamma_{t} \eta$. However, the solutions of the linear error equations can be estimated finer by

$$
\left|s_{t}\right|=\left|L_{t}^{\text {abs }} J_{u} e\right| \leqslant \sigma_{t} \eta, \quad\left|r_{t}\right|=\left|L_{t}^{\text {rel }} e\right|<\rho_{t} \eta,
$$

using the weighted absolute and relative a priori condition numbers

$$
\sigma_{t}=\sum_{k=0}^{t}\left|L_{t k}^{\mathrm{abs}} u_{k}\right| \gamma_{k}, \quad \rho_{t}=\sum_{k=0}^{t}\left|L_{t k}^{\mathrm{rel}}\right| \gamma_{k}, \quad t=0, \ldots, n
$$

In cases, for example, when $F_{t}$ is an input operation of a floating-point number represented exactly in the computer, the associated $\gamma_{t}$ may be set equal to zero. When a subtraction $F_{t}$ is performed with loss of significant figures but without rounding error, we may put $\gamma_{t}=0$. If a built-in function $F_{t}=f_{t}$ is evaluated in lower precision than the arithmetic operations are, the associated $\gamma_{t}$ may be chosen suitably greater than 1 . In particular, the behavior of the algorithm under data perturbations only and under rounding errors in the 'built-in' functions and arithmetic operations only can be analyzed. For this purpose, to a given sequence of weights $\left(\gamma_{t}\right)$ of the local error distribution, put

$$
\gamma_{t}^{D}=\gamma_{t}, \quad F_{t} \in(\mathrm{F} 0) ; \quad \gamma_{t}^{R}=\gamma_{t}, \quad F_{t} \in(\mathrm{F} 1) \cup(\mathrm{F} 2) ;
$$

and $\gamma_{t}^{D}, \gamma_{t}^{R}=0$ else for $t=0, \ldots, n$. The sequences of weights $\left(\gamma_{t}^{D}\right),\left(\gamma_{t}^{R}\right)$ specify, by (17), weighted absolute and relative data condition numbers $\sigma_{t}^{D}, \rho_{t}^{D}$ and rounding condition numbers $\sigma_{t}{ }^{R}, \rho_{t}{ }^{R}$. In this way, the decompositions

$$
\gamma_{t}=\gamma_{t}^{D}+\gamma_{t}^{R}, \quad \sigma_{t}=\sigma_{t}^{D}+\sigma_{t}^{R}, \quad \rho_{t}=\rho_{t}^{D}+\rho_{t}^{R},
$$

are obtained. The following corollary states error estimates using weighted condition numbers.

(19) Let any nontrivial sequence of weights $\gamma_{0}, \ldots, \gamma_{n}$ be given. For every sequence of local errors in the distribution (15) and sufficiently small $\varepsilon=\gamma \eta$ the solutions of the 
perturbed algorithm $(\tilde{\mathrm{A}})$ then satisfy the error estimates

$$
\left|\Delta u_{t}\right|<\sigma_{t} \eta+\sigma_{t}^{1} \omega_{t} \gamma^{2} \eta^{2}, \quad\left|P u_{t}\right|<\rho_{t} \eta+\rho_{t}^{1} \omega_{t} \gamma^{2} \eta^{2}, \quad t=0, \ldots, n .
$$

These estimates are optimal in the sense that for each $j$ there exists a sequence $\left(e_{t}\right)$ in (15) and an associated sequence $\left(v_{t}\right)$ of solutions of $(\tilde{\mathrm{A}})$ such that

$$
\pm \Delta u_{j}=\sigma_{j} \eta+O_{j}\left(\eta^{2}\right), \quad P u_{j}=\rho_{j} \eta+O_{j}\left(\eta^{2}\right)
$$

Proof. (i) The error estimates (12i) and the inequalities (10) immediately entail the estimate (19i). For any $j$, in particular, the local rounding error distribution

$$
e_{k}=\eta \gamma_{k} \operatorname{sgn}\left(L_{j k}^{\mathrm{rel}}\right), \quad k=0, \ldots, n,
$$

may be chosen. Thus the solutions of the linear error equations become

$$
s_{j}=u_{j} r_{j}= \pm \sigma_{j} \eta, \quad r_{j}=\sum_{k=0}^{t} L_{j k}^{\mathrm{rel}} e_{k}=\rho_{j} \eta .
$$

For sufficiently small $\eta$ the so perturbed algorithm $(\tilde{\mathrm{A}})$ is well defined. That is, there exists an associated solution $v=\left(v_{0}, \ldots, v_{n}\right)$ of $(\tilde{\mathrm{A}})$ satisfying the error estimates (12i). This yields

$$
\left| \pm \Delta u_{j}-\sigma_{j} \eta\right| \leqslant \sigma_{j}^{1} \omega_{j} \gamma^{2} \eta^{2}, \quad\left|P u_{j}-\rho_{j} \eta\right| \leqslant \rho_{j}^{1} \omega_{j} \gamma^{2} \eta^{2}
$$

whence (19ii) follows.

Before we continue our study let us first define the notion of sensitivity of a solution $z$ of a given problem with respect to data perturbations. Let the solution $z$ be a function $Z$ of $m$ parameters in a neighborhood $U$ of a data vector $c=$ $\left(c_{1}, \ldots, c_{m}\right)$. Let $Z$ be Fréchet-differentiable at the point $c$ and let $c_{i} \neq 0$ for $i=1, \ldots, m$. Then, for sufficiently small $\eta$, all data vectors $c^{\prime}=\left(c_{1}^{\prime}, \ldots, c_{m}^{\prime}\right)$ having the property

$$
\left|P c_{i}\right| \leqslant \gamma_{i}^{c} \eta, \quad i=1, \ldots, m,
$$

belong to the neighborhood $U$. The constants $\gamma_{1}^{c}, \ldots, \gamma_{m}^{c}$ are positive weights. Obviously, the error

$$
\Delta z=Z\left(c^{\prime}\right)-Z(c)=Z^{\prime}(c) J_{c} P c+o(\eta)
$$

is bounded, optimally, by

$$
|\Delta z| \leqslant \sum_{i=1}^{m}\left|\frac{\partial Z}{\partial c_{i}}(c) c_{i}\right| \gamma_{i}^{c} \eta+o(\eta)
$$

for all $c^{\prime}$ in the neighborhood (20) of $c$. The factor of $\eta$ on the right-hand side of the last inequality is an asymptotic absolute condition $\left\|Z^{\prime}(c)\right\|$ of the function $Z$ in the sense of Rice [15] if $U$ is equipped with the norm

$$
\|h\|=\max _{i=1, \ldots, m} \frac{1}{\gamma_{i}^{c}}\left|\frac{h_{i}}{c_{i}}\right|, \quad h=\left(h_{1}, \ldots, h_{m}\right) .
$$

Now choose any algorithm of the kind that is considered in this paper for computing the solution of the given problem such that under data perturbations only, assuming exact 'built-in' functions and arithmetic operations,

$$
u_{t_{i}}=c_{i}, \quad v_{t_{i}}=c_{i}^{\prime}, \quad \gamma_{t_{i}}^{D}=\gamma_{i}^{c}, \quad i=1, \ldots, m,
$$


$\gamma_{t}^{D}=0$ for $t \notin\left\{t_{1}, \ldots, t_{m}\right\}$, and $Z(c)=u_{t}, Z\left(c^{\prime}\right)=v_{t}$ for all $c^{\prime}$ in the neighborhood (20) of $c$. Thus, on the one hand (21) holds and on the other hand, by (3), (14),

$$
\Delta u_{t}=L_{t}^{\mathrm{abs}} J_{u} e^{D}+O_{t}\left(\eta^{2}\right)
$$

where $e_{t_{i}}^{D}=P c_{i}, e_{t}^{D}=0$ for $t \notin\left\{t_{1}, \ldots, t_{m}\right\}$. Consequently,

$$
L_{t}^{\text {abs }} J_{u} e^{D}=Z^{\prime}(c) J_{c} P c+o(\eta)
$$

for all $P c_{i}=e_{t_{i}}^{D}$ in (20). From this relation one readily concludes that the coefficients of the two linear forms coincide and, consequently,

$$
\sigma_{t}^{D}=\sum_{k=0}^{t}\left|L_{t k}^{\mathrm{abs}} u_{k}\right| \gamma_{k}^{D}=\sum_{i=1}^{m}\left|\frac{\partial Z}{\partial c_{i}}(c) c_{i}\right| \gamma_{i}^{c} .
$$

This result shows that the value of $\sigma_{t}^{D}$ is independent of the special evaluation algorithm and a measure of the data sensitivity of the solution of the given problem.

The well-known backward analysis represents perturbations of the algorithm under rounding errors by means of data perturbations. This procedure may now be specified quantitatively as follows. Error distributions (15) with the weights $\left(\gamma_{t}^{R}\right)$ and accuracy constant $\eta=\eta_{R}$ induce perturbations of function evaluations and arithmetic operations only, the data remain unperturbed. By Theorem (12) and (16), (17), the absolute errors $\left(\Delta u_{t}\right)^{R}=v_{t}^{R}-u_{t}$ of the sequence $\left(v_{t}^{R}\right)$ of solutions of the so perturbed algorithm satisfy the relations

$$
\left(\Delta u_{t}\right)^{R}=s_{t}^{R}+O_{t}\left(\eta_{R}^{2}\right), \quad\left|s_{t}^{R}\right| \leqslant \sigma_{t}^{R} \eta_{R}
$$

For any index $j$ such that $\sigma_{j}^{D} \neq 0$ choose the data accuracy

$$
\eta_{D}=\frac{\sigma_{j}^{R}}{\sigma_{j}^{D}} \eta_{R}=\frac{\rho_{j}^{R}}{\rho_{j}^{D}} \eta_{R} .
$$

Under data perturbations only, the associated linear forms $L_{j}^{\text {abs }} J_{u} e^{D}$ then have the closed intervals

$$
\left[-\sigma_{j}^{D} \eta_{D},+\sigma_{j}^{D} \eta_{D}\right]=\left[-\sigma_{j}^{R} \eta_{R},+\sigma_{j}^{R} \eta_{R}\right]
$$

as their range. Hence, $s_{j}^{R}$ belongs to this range and there exists a sequence $e^{D}$ of

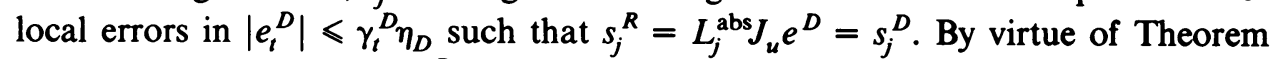
(12), using this sequence $e^{D}$ of local errors and the accuracy constant $\eta_{D}$, there exists a sequence $\left(v_{t}^{D}\right)$ of solutions of the algorithm under data perturbations only such that $\left(\Delta u_{t}\right)^{D}=s_{t}{ }^{D}+O_{t}\left(\eta_{D}^{2}\right)$. It follows from the above that $\eta_{D}$ is the least constant such that the absolute error $\left(\Delta u_{j}\right)^{D}=v_{j}^{D}-u_{j}$ gives the representation $\left(\Delta u_{j}\right)^{R}=\left(\Delta u_{j}\right)^{D}+O_{j}\left(\eta_{R}^{2}\right)$ for arbitrary local rounding errors $e_{t}^{R}$ in $\left|e_{t}^{R}\right|<\gamma_{t}^{R} \eta_{R}$. Consequently, the constant $\sigma_{j}^{R} / \sigma_{j}{ }^{D}=\rho_{j}^{R} / \rho_{j}{ }^{D}$ measures the stability of the algorithm for computing $u_{j}$ in the sense of Wilkinson's backward error analysis [25, I-39].

An algorithm is called by Bauer [4], [5] well-conditioned (German: gutartig) if the influence of rounding errors in the arithmetic operations is at most of the same order of magnitude as the influence of data perturbations whose magnitude 
corresponds to rounding errors. Thus the computation of $u_{t}$ by the algorithm (A) is well-conditioned if the estimates

$$
\sigma_{t}^{R} \leqslant \beta \sigma_{t}^{D}, \quad \rho_{t}^{R}<\beta \rho_{t}^{D}
$$

hold where the constant $\beta$ is not much greater than 1 .

Example 1. The product algorithm

$$
u_{0}=b_{0}, \quad u_{t}=b_{t} u_{t-1}, \quad t=1, \ldots, n,
$$

for the computation of $u_{n}=\prod_{t=0}^{n} b_{t}$ possesses the condition numbers

$$
\rho_{t}^{D}=t+1, \quad \rho_{t}^{R}=t .
$$

Hence $\rho_{t}^{R} \leqslant \rho_{t}^{D}$, so that this algorithm is well-conditioned.

Example 2. The summation algorithm

$$
u_{0}=c_{0}, \quad u_{t}=u_{t-1}+c_{t}, \quad t=1, \ldots, n,
$$

for the computation of the sequence of partial sums $u_{t}=\sum_{k=0}^{t} c_{k}, t=0, \ldots, n$, has the condition numbers

$$
\sigma_{t}^{D}=\sum_{j=0}^{t}\left|c_{j}\right|, \quad \sigma_{t}^{R}=\sum_{j=1}^{t}\left|u_{j}\right| .
$$

In the case of nonnegative terms it follows that

$$
\rho_{t}^{D}=1, \quad \rho_{t}^{R}=\frac{1}{u_{t}} \sum_{j=1}^{t} u_{j} \leqslant t .
$$

Consequently, the summation algorithm for the computation of $u_{n}$ is wellconditioned if and only if $\rho_{n}^{R}$ is not much greater than 1 , so that the relative error $P u_{n}$ is bounded by a low multiple of the floating-point accuracy constant $\eta$. This condition is not true in many cases. A well-known concrete example is the summation of $c_{j}=h$ for $j=0, \ldots, n$. In this case

$$
u_{t}=(t+1) h, \quad \rho_{t}^{R}=\frac{t+3}{t+1} \cdot \frac{t}{2}, \quad t=1, \ldots, n .
$$

For

$$
h=.555, \quad n=200, \quad u_{n}=111.555, \quad \rho_{n}^{R} \doteq 101,
$$

in 3-digit decimal floating point $v_{n}=133, P u_{n} \doteq .192$, is computed. Here $\eta=$ 5. $-03, \rho_{n}^{R} \eta=.505$, so that this bound overestimates the error only by a factor of 2.63 .

2.2. A Posteriori Condition Numbers and Error Estimates. In many examples the condition numbers of the algorithm or appropriate bounds can be computed from the data and intermediate results arising during the computation. For this purpose the a posteriori condition numbers are needed, being specified by the sequence of solutions $v_{0}, \ldots, v_{n}$ of the perturbed algorithm $(\tilde{\mathrm{A}})$. The linear a posteriori error equations 1.3(16) yield, together with the solution operators $1.3(24)$, the approximations

$$
s_{t}=L_{t}^{\mathrm{abs}} J_{v} e^{\prime}, \quad r_{t}=L_{t}^{\mathrm{rel}} e^{\prime}
$$


of the a posteriori errors $\Delta v_{t}, P v_{t}$. The associated weighted a posteriori condition numbers

$$
\sigma_{t}=\sum_{k=0}^{t}\left|L_{t k}^{\mathrm{abs}} v_{k}\right| \gamma_{k}, \quad \rho_{t}=\sum_{k=0}^{t}\left|L_{t k}^{\mathrm{rel}}\right| \gamma_{k}
$$

are optimal bounds in the estimates

$$
\left|s_{t}\right| \leqslant \sigma_{t} \eta^{\prime}, \quad\left|r_{t}\right| \leqslant \rho_{t} \eta^{\prime}
$$

for all local relative a posteriori errors $\left|e_{k}^{\prime}\right| \leqslant \gamma_{k} \eta^{\prime}, k=0, \ldots, n$. In view of 1.3(25),

$$
s_{t}=v_{t} r_{t}, \quad \sigma_{t}=\left|v_{t}\right| \rho_{t} .
$$

The aim of the following investigation is to compare the solutions of the linear a priori and a posteriori error equations as well as the a priori and a posteriori condition numbers. For distinctness the upper index $i$ indicates a priori terms from Section 2.1 and the upper index 0 a posteriori terms defined above. In addition, the nonweighted a priori condition numbers

$$
\sigma_{t}^{1}=\sum_{k=0}^{t}\left|L_{t k}^{i} u_{k}\right|, \quad \rho_{t}^{1}=\frac{\sigma_{t}^{1}}{\left|u_{t}\right|}, \quad t=0, \ldots, n,
$$

are used in error estimates, $L^{i}$ denoting the absolute a priori solution operator $\left(A^{\prime} u\right)^{-1}$.

The first lemma is basic for the following. It shows that the derivatives $F^{\prime}, A^{\prime}=$ $I-F^{\prime}$ are Lipschitz continuous at $u$.

(6) There exist constants $\zeta_{t}$ such that for all local errors $\left|e_{k}\right| \leqslant \varepsilon, k=0, \ldots, n$, and sufficiently small $\varepsilon$ the following inequalities are valid:

$$
\sum_{k=0}^{t-1}\left|\frac{u_{k}}{u_{t}}\left(\frac{\partial F_{t}}{\partial x_{k}}(v)-\frac{\partial F_{t}}{\partial x_{k}}(u)\right)\right| \leqslant \zeta_{t} \max _{k<t}\left|P u_{k}\right|, \quad t=0, \ldots, n .
$$

Proof. (i) For those $t$ for which $F_{t}$ is an input operation, $\partial F_{t} / \partial x_{k}=0$, so that the above inequality holds trivially with $\zeta_{t}=0$. For additions and subtractions $F_{t}$, the partial derivatives $\partial F_{t} / \partial x_{k}$ are constant and equal to $0,+1,-1$ for each $k$. Hence for these $t$ the left side in (6i) vanishes, and the inequality is true too with $\zeta_{t}=0$.

(ii) Next consider the indices $t$ such that $F_{t}$ is a multiplication. As is readily seen, now

$$
\sum_{k=0}^{t-1}\left|\frac{u_{k}}{u_{t}}\left(\frac{\partial F_{t}}{\partial x_{k}}(v)-\frac{\partial F_{t}}{\partial x_{k}}(u)\right)\right|=\left|P u_{i_{i}}\right|+\left|P u_{j_{t}}\right|
$$

whence (6i) follows using the constant $\zeta_{t}=2$.

(iii) By virtue of Theorem 2.1(12), there exists an $\varepsilon_{1}$ such that for all $\varepsilon \leqslant \varepsilon_{1}$ the solutions $v_{t}$ of the perturbed algorithm $(\tilde{\mathrm{A}})$ are in the neighborhoods defined by 1.2(12i). In particular, then $\left|P u_{t}\right| \leqslant \frac{1}{3}$ for all $t$. One easily verifies the estimate

$$
\sum_{k=0}^{t-1}\left|\frac{u_{k}}{u_{t}}\left(\frac{\partial F_{t}}{\partial x_{k}}(v)-\frac{\partial F_{t}}{\partial x_{k}}(u)\right)\right|<\frac{2\left|P u_{j}\right|}{\left|1+P u_{j}\right|}+\frac{\left|P u_{j}-P u_{i}\right|}{\left|1+P u_{j}\right|^{2}}
$$

for divisions $F_{t}(x)=x_{i} / x_{j}$. As $\left|P u_{j}\right|<\frac{1}{3}$, the denominators are bounded from below by $\frac{2}{3}$ and $\frac{4}{9}$. Thus the inequality (6i) holds in this case with $\zeta_{t}=7.5$. 
(iv) In function evaluations $F_{t}(x)=f_{t}\left(x_{i}\right), i=i_{t}$, finally,

$$
\sum_{k=0}^{t-1}\left|\frac{u_{k}}{u_{t}}\left(\frac{\partial F_{t}}{\partial x_{k}}(v)-\frac{\partial F_{t}}{\partial x_{k}}(u)\right)\right|=\left|\frac{u_{i}^{2}}{f_{t}\left(u_{i}\right)} f_{t}^{\prime \prime}\left(w_{i}\right) P u_{i}\right|
$$

$w_{i}$ being some intermediate point of the line segment $\bar{u}_{i} v_{i}$. By Theorem 1.2(12), the join $\overline{u v}$ belongs to the domain of definition of $F^{\prime}$. The right-hand side of the last equation is bounded by $2 \kappa_{t}\left|P u_{i}\right|$, using the constants $\kappa_{t}$ of Theorem 1.2(12). Consequently, (6i) is true for $\zeta_{t}=2 \kappa_{t}$.

By the above lemma, we can now estimate the distance between the a priori and a posteriori solution operators

$$
L^{i}=\left(A^{\prime} u\right)^{-1}, \quad L^{0}=\left(A^{\prime} v\right)^{-1} .
$$

(8) There exists a constant $\mu$ such that for all local errors $\left|e_{k}\right|<\varepsilon, k=0, \ldots, n$, and sufficiently small $\varepsilon$ the associated solution operators suffice the following estimates

$$
\sum_{k=0}^{t}\left|L_{t k}^{0}-L_{t k}^{i}\right|\left|u_{k}\right| \leqslant \sigma_{t}^{1} \mu \varepsilon, \quad t=0, \ldots, n .
$$

Proof. (i) As is readily verified, the difference of the solution operators has the representation

$$
L^{0}-L^{i}=-L^{i}(I+C)^{-1} C,
$$

where $C=\left(A^{\prime} v-A^{\prime} u\right) L^{i}=\left(F^{\prime}(u)-F^{\prime}(v)\right) L^{i}$. This implies

$$
\left(L^{0}-L^{i}\right) J_{u}=-L^{i} J_{u}(I+D)^{-1} D,
$$

using the notation

$$
D=J_{u}^{-1} C J_{u}=J_{u}^{-1}\left(F^{\prime}(u)-F^{\prime}(v)\right) L^{i} J_{u} .
$$

Let us further denote by $J_{\sigma}$ the diagonal matrix $\operatorname{diag}\left(\sigma_{0}^{1}, \ldots, \sigma_{n}^{1}\right)$. In the maximum absolute row sum norm then

$$
\left\|J_{\sigma}^{-1} L^{i} J_{u}\right\|=\max _{t} \frac{1}{\sigma_{t}^{1}} \sum_{k=0}^{t}\left|L_{t k}^{i} u_{k}\right|=1
$$

The matrix $D$ is bounded in this norm by

$$
\|D\| \leqslant\left\|J_{u}^{-1}\left(F^{\prime}(u)-F^{\prime}(v)\right) J_{\sigma}\right\|=\max _{t} \sum_{k=0}^{t-1}\left|\frac{u_{k}}{u_{t}}\left(\frac{\partial F_{t}}{\partial x_{k}}(v)-\frac{\partial F_{t}}{\partial x_{k}}(u)\right)\right| \rho_{k}^{1} .
$$

From Lemma (6) we infer the further estimate

$$
\|D\| \leqslant \zeta \rho \max _{t}\left|P u_{t}\right|, \quad \zeta=\max _{t} \zeta_{t}, \quad \rho=\max _{t} \rho_{t}^{1} .
$$

Now choose any constant $\vartheta$ in $0<\vartheta<1$ and put

$$
\tau=(1+\vartheta) \rho, \quad \varepsilon \leqslant \min \left(\varepsilon_{1}, \frac{\vartheta}{\omega}, \frac{\vartheta}{\zeta \rho \tau}\right), \quad \omega=\max _{t} \omega_{t}
$$

Theorem 2.1(12) then guarantees that

$$
\max _{t}\left|P u_{t}\right| \leqslant(1+\omega \varepsilon) \rho \varepsilon \leqslant \tau \varepsilon
$$


The above estimate (11) hereby yields $\|D\| \leqslant \zeta \rho \tau \varepsilon \leqslant \vartheta<1$. Finally (9), (10), (11) entail the estimate

$$
\left\|J_{\sigma}^{-1}\left(L^{0}-L^{i}\right) J_{u}\right\|<\frac{\|D\|}{1-\|D\|}<\mu \varepsilon,
$$

using the constant $\mu=\zeta \rho \tau /(1-\vartheta)$, and thence the asserted inequality (8i).

Having made these preparations, we are in the position to prove the comparison theorem. By $s^{i}, r^{i}$ are meant the solutions of the linear a priori error equations 1.3(15), and by $s^{0}, r^{0}$ the solutions of the linear a posteriori error equations 1.3(16). Further, $\sigma_{t}^{i}, \rho_{t}^{i}$ denote the weighted a priori condition numbers 2.1(17) and $\sigma_{t}^{0}, \rho_{t}^{0}$ the a posteriori condition numbers (2).

(12) For all local errors $\left|e_{k}\right| \leqslant \gamma_{k} \eta, k=0, \ldots, n$, and sufficiently small $\eta$ the approximations of the absolute and relative a priori and a posteriori errors satisfy the relations

$$
s_{t}^{0}=-s_{t}^{i}+O_{t}\left(\eta^{2}\right), \quad r_{t}^{0}=-r_{t}^{i}+O_{t}\left(\eta^{2}\right),
$$

and the associated weighted condition numbers the relations

$$
\sigma_{t}^{0}=\sigma_{t}^{i}+O_{t}(\eta), \quad \rho_{t}^{0}=\rho_{t}^{i}+O_{t}(\eta)
$$

Proof. (i) The solutions of the linear absolute a priori and a posteriori error equations have the representations $s^{i}=L^{i} J_{u} e, s^{0}=L^{0} J_{v} e^{\prime}$. Thus,

$$
s_{t}^{i}+s_{t}^{0}=L_{t}^{i}\left(J_{u}-J_{v}\right) e+L_{t}^{i} J_{v}\left(e+e^{\prime}\right)+\left(L_{t}^{0}-L_{t}^{i}\right) J_{v} e^{\prime} .
$$

The first term on the right side is bounded by

$$
\left|L_{t}^{i}\left(J_{u}-J_{v}\right) e\right| \leqslant \sum_{k=0}^{t}\left|L_{t k}^{i} u_{k} P u_{k} e_{k}\right| \leqslant \sigma_{t}^{i}\|P u\| \eta
$$

using the maximum norm for $P u$. By virtue of $1.1(8), e_{k}+e_{k}^{\prime}=-e_{k} e_{k}^{\prime}$. For $\left|e_{k}\right| \leqslant \gamma_{k} \eta$ it follows that

$$
\left|e_{k}^{\prime}\right|=\left|\frac{e_{k}}{1+e_{k}}\right| \leqslant \frac{\gamma_{k} \eta}{1-\gamma \eta} \leqslant \gamma^{\prime} \eta, \quad \gamma=\max \gamma_{k}, \quad \gamma^{\prime}=\frac{\gamma}{1-\gamma \eta} .
$$

Therefore, the second term on the right side suffices

$$
\left|L_{t}^{i} J_{v}\left(e+e^{\prime}\right)\right| \leqslant \sum_{k=0}^{t}\left|L_{t k}^{i} u_{k} \frac{v_{k}}{u_{k}} e_{k} e_{k}^{\prime}\right| \leqslant \sigma_{t}^{i}(1+\|P u\|) \gamma^{\prime} \eta^{2}
$$

By virtue of Lemma (8) for $\varepsilon=\gamma \eta$, the third term can finally be estimated by

$$
\left|\left(L_{t}^{0}-L_{t}^{i}\right) J_{v} e^{\prime}\right| \leqslant \sum_{k=0}^{t}\left|\left(L_{t k}^{0}-L_{t k}^{i}\right) u_{k} \frac{v_{k}}{u_{k}} e^{\prime}\right| \leqslant \sigma_{t}^{1} \mu \gamma(1+\|P u\|) \gamma^{\prime} \eta^{2} .
$$

On choosing $\varepsilon$ as small as in the proof of Lemma (8), we have $\|P u\|<\tau \varepsilon$ and $\|P u\| \leqslant \frac{1}{3}$. The above appraisals then entail $\left|s_{t}^{i}+s_{t}^{0}\right|<\left(\sigma_{t}^{i} \phi+\sigma_{t}^{1} \psi\right) \eta^{2}$, using the constants

$$
\phi=\tau \gamma+\frac{4}{3} \gamma^{\prime}, \quad \psi=\frac{4}{3} \mu \gamma \gamma^{\prime} .
$$

(ii) The solutions $r^{i}, r^{0}$ of the linear relative a priori and a posteriori error equations permit the representation

$$
r_{t}^{i}+r_{t}^{0}=\frac{1}{1+P u_{t}}\left\{\frac{s_{t}^{i}+s_{t}^{0}}{u_{t}}+r_{t}^{i} P u_{t}\right\}
$$


For $\gamma \eta=\varepsilon \leqslant \varepsilon_{1}$, again $\left|P u_{t}\right| \leqslant \tau \varepsilon$ and $\left|P u_{t}\right| \leqslant \frac{1}{3}$. Using the above estimates for $s_{t}^{i}+s_{t}^{0}$ and 2.1(16), thus

$$
\frac{2}{3}\left|r_{t}^{i}+r_{t}^{0}\right|<\left(\rho_{t}^{i} \phi+\rho_{t}^{1} \psi\right) \eta^{2}+\rho_{t}^{i} \tau \gamma \eta^{2} .
$$

(iii) The weighted absolute condition numbers satisfy the relations

$$
\begin{aligned}
\frac{1}{\gamma}\left|\sigma_{t}^{i}-\sigma_{t}^{0}\right| & \leqslant \sum_{k=0}^{t}\left|L_{t k}^{i} u_{k}-L_{t k}^{0} v_{k}\right| \\
& \leqslant \sum_{k=0}^{t}\left|L_{t k}^{i} u_{k} P u_{k}\right|+\sum_{k=0}^{t}\left|\left(L_{t k}^{0}-L_{t k}^{i}\right) v_{k}\right|
\end{aligned}
$$

The first term on the right side can be estimated by

$$
\sum_{k=0}^{t}\left|L_{t k}^{i} u_{k} P u_{k}\right|<\sigma_{t}^{1}\|P u\| \leqslant \sigma_{t}^{1} \tau \gamma \eta
$$

Using Lemma (8), the second term possesses the upper bound

$$
\sum_{k=0}^{t}\left|\left(L_{t k}^{0}-L_{t k}^{i}\right) u_{k}\left(1+P u_{k}\right)\right| \leqslant \sigma_{t}^{1} \mu \varepsilon(1+\|P u\|) .
$$

Hence, for $\varepsilon=\gamma \eta \leqslant \varepsilon_{1}$, the above yields

$$
\left|\sigma_{t}^{i}-\sigma_{t}^{0}\right|<\sigma_{t}^{1} \chi \eta, \quad \chi=\left(\tau+\frac{4}{3} \mu\right) \gamma
$$

(iv) Finally, the difference of the weighted relative a priori and a posteriori condition numbers is bounded by

$$
\left|\rho_{t}^{i}-\rho_{t}^{0}\right| \leqslant \frac{1}{\left|1+P u_{t}\right|}\left\{\frac{\left|\sigma_{t}^{i}-\sigma_{t}^{0}\right|}{\left|u_{t}\right|}+\rho_{t}^{i}\left|P u_{t}\right|\right\} .
$$

Together with the estimate for $\left|\sigma_{t}^{i}-\sigma_{t}^{0}\right|$ this entails

$$
\frac{2}{3}\left|\rho_{t}^{i}-\rho_{t}^{0}\right| \leqslant \rho_{t}^{1} \chi \eta+\rho_{t}^{i} \tau \gamma \eta
$$

In computing a posteriori condition numbers or solutions of linear a posteriori error equations, Table 1.2 yields modified coefficients. The modified relative a posteriori error equations $\tilde{r}-\tilde{B} \tilde{r}=e^{\prime}$ for approximations $\tilde{r}$ of $P v$ are defined by matrix elements $\tilde{b}_{t k}$ of $\tilde{B}$ of the form 1.2(27), (28),

(13) $\quad F_{t}=+,-: \quad \tilde{b}_{t k}=\frac{v_{k}}{v_{t}} \frac{\partial F_{t}}{\partial x_{k}}(v) ; \quad F_{t}=\times, /: \quad \tilde{b}_{t k}=\frac{v_{k}}{F_{t}(v)} \frac{\partial F_{t}}{\partial x_{k}}(v)$,

whereas the elements $b_{t k}$ of the unmodified relative a posteriori error equations $r-B r=e^{\prime}$, by $1.3(18)$, read

$$
b_{t k}=\frac{v_{k}}{v_{t}} \frac{\partial F_{t}}{\partial x_{k}}(v)
$$

We limit the further study to the four arithmetic operations,,$+- \times, /$ and establish the theorem

(15) The approximations $r_{t}, \tilde{r}_{t}$ of the relative a posteriori errors $P v_{t}$ satisfy, for sufficiently small $\varepsilon=\gamma \eta$, the relations

$$
\tilde{r}_{t}=r_{t}+O_{t}\left(\eta^{2}\right)
$$

and the associated weighted relative a posteriori condition numbers the relations

$$
\tilde{\rho}_{t}=\left(1+O_{t}(\eta)\right) \rho_{t}, \quad t=1, \ldots, n .
$$


Proof. (i) The two equations for $r, \tilde{r}$ immediately imply the representation

$$
\tilde{r}-r=L \Delta B \tilde{r}, \quad L=\left(A^{\prime} v\right)_{\mathrm{rel}}^{-1}, \quad \Delta B=\tilde{B}-B .
$$

When $F_{k}=+,-, \Delta b_{k l}=0$, whereas for $F_{k}=\times, /$ we obtain

$$
\Delta b_{k l}=\tilde{b}_{k l}-b_{k l}=-e_{k}^{\prime} \tilde{b}_{k l}
$$

because $F_{k}(v) / v_{k}=1+e_{k}^{\prime}$. The above leads to the estimate

$$
\left|\tilde{r}_{t}-r_{t}\right| \leqslant \eta^{\prime} \sum_{\substack{k=0 \\ F_{k}=\times, /}}^{t}\left|L_{t k}^{\mathrm{rel}}\right| \gamma_{k} \sum_{l=0}^{k-1}\left|\tilde{b}_{k l} \tilde{r}_{l}\right|<2 \eta^{\prime} \rho_{t} \max _{j<t}\left|\tilde{r}_{j}\right|
$$

because $\left|\tilde{b}_{k l}\right|=1$ exactly twice and zero else for $F_{k}=\times, /$. Further, the last inequality yields

$$
\max _{j<t}\left|\tilde{r}_{j}\right| \leqslant \rho_{t}^{\prime} \eta^{\prime}+2 \eta^{\prime} \rho_{t}^{\prime} \max _{j<t}\left|\tilde{r}_{j}\right|
$$

using the constants $\rho_{t}^{\prime}=\max _{j<t} \rho_{j}, \eta^{\prime}=\eta /(1-\gamma \eta)$. Hence

$$
\max _{j<t}\left|\tilde{r}_{j}\right|<\frac{\rho_{t}^{\prime}}{1-2 \rho_{t}^{\prime} \eta} \eta^{\prime}
$$

as far as $2 \rho_{t}^{\prime} \eta^{\prime}<1$. Inserting this expression into the above inequality for $\tilde{r}_{t}-r_{t}$, we obtain

$$
\left|\tilde{r}_{t}-r_{t}\right| \leqslant \frac{2 \rho_{t}^{\prime} \rho_{t}}{1-2 \rho_{t}^{\prime} \eta^{\prime}} \eta^{\prime 2}
$$

and thus the assertion (15i) is proved.

(ii) Next, using appropriate constants $\psi_{t}$,

$$
\left|\tilde{r}_{t}\right| \leqslant\left|r_{t}\right|+\rho_{t} \psi_{t} \eta^{2}, \quad\left|r_{t}\right|<\left|\tilde{r}_{t}\right|+\rho_{t} \psi_{t} \eta^{2} .
$$

The associated weighted relative a posteriori condition numbers $\rho_{t}, \tilde{\rho}_{t}$, permit the estimate $\left|\tilde{r}_{t}\right|<\tilde{\rho}_{t} \eta^{\prime},\left|r_{t}\right| \leqslant \rho_{t} \eta^{\prime}$, for all local errors $\left|e_{k}\right|<\gamma_{k} \eta$ where $\gamma=\max \gamma_{k}$ and $\eta^{\prime}$ as above. By means of the local errors

$$
\tilde{e}_{k}=-\gamma_{k} \eta \operatorname{sgn}\left(\tilde{L}_{t k}^{\mathrm{rel}}\right), \quad e_{k}=-\gamma_{k} \eta \operatorname{sgn}\left(L_{t k}^{\mathrm{rel}}\right),
$$

we finally conclude

$$
\frac{\tilde{\rho}_{t}}{1+\gamma \eta} \leqslant \frac{\rho_{t}}{1-\gamma \eta}+\rho_{t} \psi_{t} \eta, \quad \frac{\rho_{t}}{1+\gamma \eta} \leqslant \frac{\tilde{\rho}_{t}}{1-\gamma \eta}+\rho_{t} \psi_{t} \eta
$$

hereby proving (15ii).

By Theorems (12) and (15), we have established the a posteriori error representation

$$
\frac{u_{t}-v_{t}}{v_{t}}=\tilde{r}_{t}+O_{t}\left(\eta^{2}\right)
$$

where $\tilde{r}$ is the solution of the modified linear relative a posteriori error equations. Solving this relation for $u_{t}$ yields

$$
u_{t}=v_{t}\left(1+\tilde{r}_{t}+O_{t}\left(\eta^{2}\right)\right)
$$

Hence

$$
v_{t}^{\prime}=v_{t}\left(1+\tilde{r}_{t}\right)
$$


is an approximation of $u_{t}$ having the relative error $\left(u_{t}-v_{t}^{\prime}\right) / v_{t}^{\prime}=O_{t}\left(\eta^{2}\right)$. Consequently, $v_{t}^{\prime}$ approximates $u_{t}$ within about double precision. The sequence of solutions $u_{0}, \ldots, u_{n}$ of the algorithm (A) may thus be computed in about double precision from the approximations $v_{t}$ and the solutions $\tilde{r}_{t}$ of the modified linear a posteriori error equations, both computed in single precision. We call this procedure extrapolation to double precision. The a posteriori coefficients $b_{t k}$ are readily computed from the data and intermediate results in performing the algorithm. If the local errors $e_{t}$ are available numerically, the solutions $\tilde{r}_{t}$ of the linear a posteriori error equations and the extrapolated approximations $v_{t}^{\prime}$ can be computed together with $v_{t}$.

TABLE 2.2

Extrapolation to double precision in Cramer's rule

\begin{tabular}{|c|c|c|c|c|c|}
\hline$t$ & $u_{t}$ & $v_{t}$ & $e_{t}$ & $-\tilde{r}_{t} \times 10^{3}$ & \\
\hline 0 & $a$ & 0.455 & $1.00-03$ & 1.00 & \\
\hline 1 & $b$ & 0.111 & $-1.00-03$ & -1.00 & \\
\hline 2 & $c$ & 0.273 & $1.00-03$ & 1.00 & \\
\hline 3 & $d$ & 0.778 & $2.86-04$ & 0.29 & \\
\hline 4 & $f$ & 0.404 & $-1.00-04$ & -0.10 & \\
\hline 5 & $g$ & 0.566 & $6.07-04$ & 0.61 & \\
\hline 6 & $a d$ & 0.354 & $2.82-05$ & 1.31 & \\
\hline 7 & $b c$ & 0.0303 & $-9.90-05$ & -0.10 & \\
\hline 8 & $d f$ & 0.314 & $-9.93-04$ & -0.81 & \\
\hline 9 & bg & 0.0628 & $-4.14-04$ & -0.81 & \\
\hline 10 & ag & 0.258 & $1.83-03$ & 3.44 & \\
\hline 11 & $c f$ & 0.110 & $-2.65-03$ & -1.75 & \\
\hline 12 & $D_{0}$ & 0.324 & $9.27-04$ & 2.36 & \\
\hline 13 & $D_{1}$ & 0.251 & $-7.96-04$ & -1.60 & \\
\hline 14 & $D_{2}$ & 0.148 & 0 & 7.29 & \\
\hline 15 & $x$ & 0.775 & $3.98-04$ & -3.56 & $\frac{x^{\prime}-x}{x}=3.57-03$ \\
\hline 16 & $y$ & 0.457 & 4.59-04 & 5.39 & $\frac{y^{\prime}-y}{y}=5.40-03$ \\
\hline & $x=0 . \overline{7}$ & \multicolumn{3}{|c|}{$x^{\prime}=0.775$} & $\frac{x^{\prime \prime}-x}{x}=-1.13-05$ \\
\hline & $y=0 . \overline{45}$ & \multicolumn{3}{|c|}{$y^{\prime}=0.457$} & $\frac{y^{\prime \prime}-y}{y}=1.00-05$ \\
\hline
\end{tabular}

Example. Two linear equations in two unknowns,

$$
a x+b y=f, \quad c x+d y=g,
$$

have, by Cramer's rule, the solution

$$
x=\frac{D_{1}}{D_{0}}, \quad y=\frac{D_{2}}{D_{0}}, \quad\left(D_{0} \neq 0\right),
$$


using the determinants

$$
D_{0}=\left|\begin{array}{ll}
a & b \\
c & d
\end{array}\right|, \quad D_{1}=\left|\begin{array}{ll}
f & b \\
g & d
\end{array}\right|, \quad D_{2}=\left|\begin{array}{ll}
a & f \\
c & g
\end{array}\right| .
$$

Consider the numerical example

$$
\frac{5}{11} x+\frac{1}{9} y=\frac{40}{99}, \quad \frac{3}{11} x+\frac{7}{9} y=\frac{56}{99},
$$

having the solution $x=7 / 9, y=5 / 11$. Table 2.2 lists the approximations $v_{t}$ in solving Cramer's rule using 3-digit decimal floating-point arithmetic, the associated local rounding errors $e_{t}$ of input and arithmetic operations, and the approximations $\tilde{r}_{t}$ of the relative a posteriori errors described above. The numerical results show that the extrapolated results $x^{\prime \prime}, y^{\prime \prime}$ approximate $x, y$ essentially within double precision.

2.3. Associated Graphs. In typical applications of the perturbation theory, the number $n$ of steps and thus of equations in the systems 1.3(15), (16), (17) often becomes very large. However, the matrices $\left(b_{t k}\right)$ of the linear error equations are sparse; for input operations, $F_{t}$ is constant and $b_{t k}=0$ for all $k$; 'built-in' functions $F_{t}$ give $b_{t k}=0$ for all $k \neq j_{t}$; for arithmetic operations $F_{t} \in(\mathrm{F} 2), b_{t k}=0$ for all $k \neq i_{t}, j_{t}$. Hence the linear error equations have the form of inhomogeneous linear recursions of zeroth, first and second order with variable coefficients. In general, however, these equations have a complex structure because $z_{t}$ is not obtained from $z_{t-1}, z_{t-2}$ but from $z_{i}, z_{j_{i}}$. This structure can be illustrated by the associated graph (see McCracken-Dorn [11], Bauer [5]): each $t$ is assigned a point in $\mathbf{R}^{2}$, called node, and nodes $k$ are connected to the node $t$ by means of a path $\overrightarrow{k t}$ if the coefficient or weight $b_{t k}$, that is, $\partial F_{t}(u) / \partial x_{k}$ or $\partial F_{t}(v) / \partial x_{k}$ can be nonzero. In addition, every path $\overrightarrow{k t}$ is labelled by its associated weight $b_{t k}$. By convention, labels 1 may be omitted. By these means linear error equations can simply be read from the graph of the algorithm. Figure 2.3 shows the building blocks of the graphs.

The graphs in Figure 2.3 may as well be viewed as graphs of the simplest numerical algorithms analyzed in Section 1.1. For instance, let

$$
u_{0}=a, u_{1}=b, \quad u_{2}=u_{1} \circ u_{0},
$$

using an arithmetic operation $\circ \in\{+,-, \times, /\}$. In floating-point arithmetic, this algorithm is realized by

$$
v_{0}=\mathrm{fl}(a), \quad v_{1}=\mathrm{fl}(b), \quad v_{2}=\mathrm{fl}\left(v_{1} \circ v_{0}\right) .
$$

The associated linear absolute and relative a priori and a posteriori error equations and their graphs then have the form shown in Figure 2.3, where $i=0, j=1, t=2$, and $r_{0}=e_{0}=P a, r_{1}=e_{1}=P b$.

Graphs constitute important means in the study of solutions of linear systems

$$
z-B z=f \Leftrightarrow z_{t}-\sum_{k=0}^{t-1} b_{t k} z_{k}=f_{t}, \quad t=0, \ldots, n,
$$

with sparse matrices, as we will explain now. The matrix $B$ is lower triangular and its diagonal elements are zero. Thus $B$ is nilpotent and $B^{n+1}=0$. The solutions $z$ of the linear system may then be represented by the finite Neumann series

$$
z=(I-B)^{-1} f=\sum_{l=0}^{n} B^{l} f
$$




$$
u_{i} \cdot(i) u_{j} \cdot(j)
$$$$
s_{i}=u_{i} e_{i}, s_{j}=u_{j} e_{j}
$$$$
r_{i}=e_{i}, \quad r_{j}=e_{j}
$$

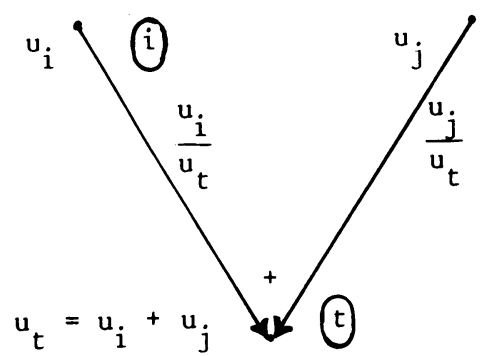

$s_{t}=s_{i}+s_{j}+u_{t} e_{t}$

$r_{t}=\frac{u_{i}}{u_{t}} r_{i}+\frac{u_{j}}{u_{t}} r_{j}+e_{t}$

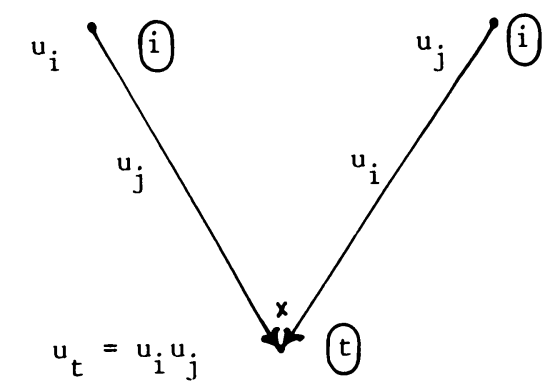

$s_{t}=u_{j} s_{i}+u_{i} s_{j}+u_{t} e_{t}$

$r_{t}=r_{i}+r_{j}+e_{t}$

(j)

$$
\begin{aligned}
& f^{\prime}\left(u_{i}\right) \\
& \frac{u_{t}}{f\left(u_{i}\right)} f^{\prime}\left(u_{i}\right) \\
& s_{t}=f^{\prime}\left(u_{i}\right) s_{i}+u_{t} e_{t} \\
& r_{t}=\frac{u_{i}}{f\left(u_{i}\right)} f^{\prime}\left(u_{i}\right) r_{i}+e_{t}
\end{aligned}
$$

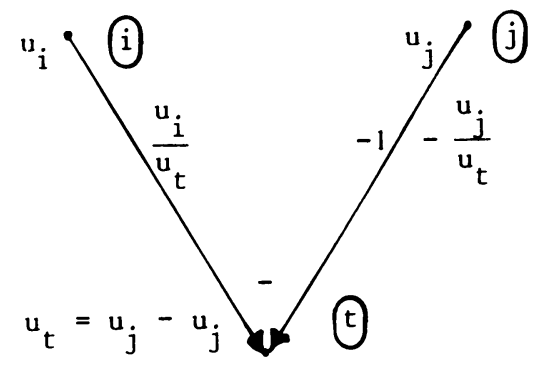

$$
\begin{aligned}
& s_{t}=s_{i}-s_{j}+u_{t} e_{t} \\
& r_{t}=\frac{u_{i}}{u_{t}} r_{i}-\frac{u_{j}}{u_{t}} r_{j}+e_{t}
\end{aligned}
$$

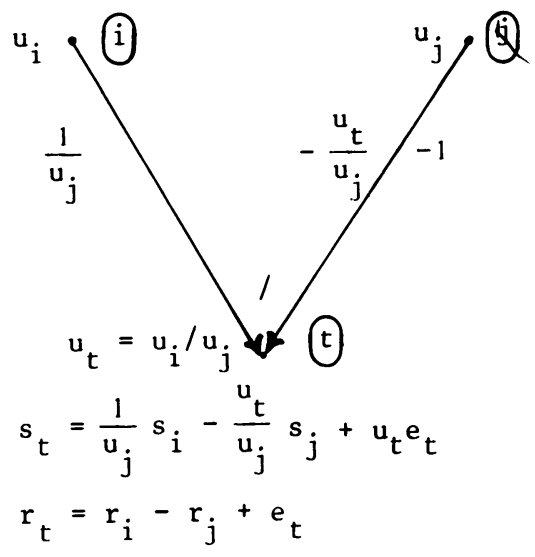

FIGURE 2.3

Building blocks of the graphs of linear absolute $\left(s_{t}\right.$, weights $b_{t k}^{\text {abs }}$ left of the path) and relative ( $r_{t}$, weights $b_{t k}^{\text {rel }}$ right of the path) a priori error equations. The associated a posteriori error equations are obtained by replacing $u_{i}, u_{j}, u_{t}, e_{t}$ by $v_{i}, v_{j}, v_{t}, e_{t}^{\prime}$. 
The powers of $B$ have the elements

$$
\begin{aligned}
& B^{0}=\left(\delta_{i k}\right), \quad B^{1}=\left(b_{i k}\right), \\
& B^{l}=\left(b_{i k}^{(l)}\right), \quad b_{i k}^{(l)}=\sum_{k_{1}=0}^{n} \cdots \sum_{k_{2}=0}^{n} b_{i k_{1}} \cdots b_{k_{2} k}, \quad i, k=0, \ldots, n,
\end{aligned}
$$

for $l=2, \ldots, n$. As $b_{i k}=0$ for $i \leqslant k$, matrix elements $b_{i k}^{(l)}$ can be different from zero only if the condition

$$
i>k_{l}>k_{l-1}>\cdots>k_{2}>k_{1}>0
$$

holds, and therefore

$$
b_{i k}^{(l)}=0 \text { for } i<k+l
$$

and all $l=1,2, \ldots$ Correspondingly, the components $z_{t}$ of the solution vector $z$ of (3) possess the representation

$$
z_{t}=L_{l} f=f_{t}+\sum_{l=1}^{t} \sum_{k=0}^{t-1} b_{t k}^{(l)} f_{k}, \quad t=0, \ldots, n .
$$

In this way, explicit representations of the components $L_{t}$ of the solution operator $L$ have been obtained. Interchanging the order of summation gives

$$
L_{0} f=f_{0}, \quad L_{k} f=\sum_{k=0}^{t} L_{t k} f_{k}=f_{t}+\sum_{k=0}^{t-1}\left(\sum_{l=1}^{t} b_{t k}^{(l)}\right) f_{k}, \quad t=1, \ldots, n .
$$

Consequently, the matrix elements of the solution operators $L=(I-B)^{-1}$ have the representation

$$
\begin{gathered}
L_{t t}=1, \quad L_{t k}=\sum_{l=1}^{t} b_{t k}^{(l)}, \quad k=0, \ldots, t-1 ; \\
L_{t k}=0, \quad k=t+1, \ldots, n .
\end{gathered}
$$

The basis of our further investigations is the explicit representation

$$
\begin{aligned}
z_{t}= & L_{t} f=f_{t}+\sum_{k_{1}=0}^{t-1} b_{t k_{1}} f_{k_{1}}+\sum_{k_{2}=0}^{t-1} \sum_{k_{1}=0}^{t-1} b_{t k_{2}} b_{k_{2} k_{1}} f_{k_{1}}+\cdots \\
& +\sum_{k_{t}=0}^{t-1} \cdots \sum_{k_{1}=0}^{t-1} b_{t k_{1}} \cdots b_{k_{2} k_{1}} f_{k_{1}},
\end{aligned}
$$

ensuing immediately from (5), (8). A sequence of paths $\vec{k}_{1} k_{2}, \ldots, \overrightarrow{k_{l} t}$ of the graph is a path of length $l$ from the node $k_{1}$ to the node $t$. We call the associated product $b_{t k_{l}} \cdots b_{k_{2} k_{1}}$ the error effect and the term $b_{t k_{1}} \cdots b_{k_{2} k_{1}} f_{k_{1}}$ the error contribution along this path at the node $t$. The error effect is simply the product of all weights along the path. On the right side of (11), evidently, only those terms of the sum can be nonzero which belong to a path of the graph, all other terms vanish. In view of (6), necessarily $t>k_{l}>\cdots>k_{2}>k_{1}$ for every path in the graph. The $l$-fold sum in (11) is the sum of the error contributions at the node $t$ along all paths of length $l$. The node $t$ itself is assigned the path or loop of length zero and the error effect 1 . The representation (11) may thus be read as follows

(12) The solution $z_{t}=L_{k} f$ of the linear error equations is the sum of the error contributions along all paths in the graph ending at the node $t$. 
On setting especially

$$
f^{(k)}=\left(\delta_{0 k}, \ldots, \delta_{n k}\right), \quad k=0, \ldots, n,
$$

as inhomogeneous terms, the associated solutions $z_{t}=L_{t} f^{(k)}$ become the matrix elements $L_{t k}$ of the solution operators $L=(I-B)^{-1}$. In particular,

$$
L_{t k}=\sum_{l=1}^{t} b_{t k}^{(l)}=b_{t k}+\sum_{k_{2}=0}^{t-1} b_{t k_{2}} b_{k_{2} k}+\cdots+\sum_{k_{t}=0}^{t-1} \cdots \sum_{k_{2}=0}^{t-1} b_{t k_{1}} \cdots b_{k_{2} k}
$$

is the total error effect associated to all paths from the node $k$ to the node $t$ (see Bauer [5, p. 88]).

Now let us consider our condition numbers in this context. The weighted absolute and relative a priori and a posteriori condition numbers 2.1(17), 2.2(2) have the general form

$$
\lambda_{t}=\sum_{k=0}^{t}\left|L_{t k}\right| \alpha_{k}
$$

where

\begin{tabular}{c|c|c}
$\lambda_{t}$ & absolute & relative \\
\hline a priori & $\sigma_{t}^{i}$ & $\rho_{t}^{i}$ \\
\hline a posteriori & $\sigma_{t}^{0}$ & $\rho_{t}^{0}$
\end{tabular}

\begin{tabular}{c|c|c}
$\alpha_{k}$ & absolute & relative \\
\hline a priori & $\left|u_{k}\right| \gamma_{k}$ & $\gamma_{k}$ \\
\hline a posteriori & $\left|v_{k}\right| \gamma_{k}$ & $\gamma_{k}$
\end{tabular}

and $L_{t k}$ denotes the matrix elements of the associated solution operators 1.3(22), (24). The condition numbers are thus weighted sums of the absolute values of the total error effects. It is readily seen from (14) that

$$
\lambda_{t}=\max _{\substack{\left|f_{k}\right|<\alpha_{k} \\ k=0, \ldots, n}}\left|L_{t} f\right|, \quad t=0, \ldots, n .
$$

The maximum is attained for $f_{k}=\alpha_{k} \operatorname{sgn} L_{t k}, k=0, \ldots, n$.

The condition numbers can be computed easily if, for instance, all matrix elements $L_{t k}$ are nonnegative. This is the case, in particular, if the elements $b_{t k}$ are nonnegative. Then $I-B$ is a so-called $M$-matrix. Under the assumption $L_{t k}>0$,

$$
\lambda_{t}=\sum_{k=0}^{t} L_{t k} \alpha_{k}=L_{t} \alpha, \quad t=0, \ldots, n,
$$

where $\alpha=\left(\alpha_{0}, \ldots, \alpha_{n}\right)$. That is, the vector $\lambda=\left(\lambda_{0}, \ldots, \lambda_{n}\right)$ of condition numbers is a solution of the linear system $\lambda-B \lambda=\alpha$ and, consequently, can be obtained recursively from

$$
\lambda_{0}=\alpha_{0}, \quad \lambda_{t}=\sum_{k=0}^{t-1} b_{t k} \lambda_{k}+\alpha_{t}, \quad t=1, \ldots, n .
$$

In the general case, where the weights $b_{t k}$ have arbitrary signs, at least bounds can be determined for the solutions $z_{t}=L_{t} f$ and thus for the corresponding condition numbers $\lambda_{i}$. The above results immediately yield the theorem:

(19) The recursion

$$
\mu_{0}=\alpha_{0}, \quad \mu_{t}=\sum_{k=0}^{t-1}\left|b_{t k}\right| \mu_{k}+\alpha_{t}, \quad t=1, \ldots, n,
$$


generates a sequence $\left(\mu_{t}\right)$ of bounds having the property

$$
\left|z_{t}\right|=\left|L_{\imath} f\right| \leqslant \mu_{t}, \quad \lambda_{t} \leqslant \mu_{t}
$$

for all $f=\left(f_{0}, \ldots, f_{n}\right)$ such that $\left|f_{k}\right| \leqslant \alpha_{k}, k=0, \ldots, n$.

When an algorithm is built up of input operations and additions only, the coefficients $b_{t k}$ of the linear absolute error equations consist, according to Table 1.2 , of zeros and ones, that is, $B$ is a binary matrix. The same is true for the linear relative error equations when the algorithm consists of input operations and multiplications only. For a binary matrix $B$, one readily sees from (13) that

$$
L_{t k}=\text { Number of paths from } k \text { to } t \text {. }
$$

The explicit representations of solution operators and associated condition numbers become very simple when the graph of the algorithm is a tree. In these graphs there exists to each $k<t$ exactly one path $\overrightarrow{k_{1} k_{2}}, \ldots, \overrightarrow{k_{s}} t$ from $k=k_{1}$ to $t$, where the length $s$ of the path depends on the pair $k, t$. Now the total error effects are simply

$$
L_{t t}=1, \quad L_{t k}=\sum_{l=1}^{t} b_{t k}^{(l)}=b_{t k_{s}} \cdots b_{k_{2} k},
$$

and the associated condition numbers have the form

$$
\lambda_{0}=\alpha_{0}, \quad \lambda_{t}=\sum_{k=0}^{t-1}\left|b_{t k_{s}}\right| \cdots\left|b_{k_{2}}\right| \alpha_{k}+\alpha_{t} .
$$

In this case, the sequence $\left(\lambda_{t}\right)$ is the solution of the recursion formulae

$$
\lambda_{0}=\alpha_{0}, \quad \lambda_{t}=\sum_{k=0}^{t-1}\left|b_{t k}\right| \lambda_{k}+\alpha_{k}, \quad t=1, \ldots, n .
$$

For, this system has the form of the linear error equations with coefficients $\left|b_{t k}\right|$ and inhomogeneous terms $\alpha_{t}$ instead of $b_{t k}, f_{t}$. According to (11), (21), the solution of the linear system (23) has just the representation (22) because the associated graph is identical with the graph of the linear error equations of the algorithm and thus also a tree.

Note the interesting fact that the condition numbers (18), (23) are obtained in the same way as those of the elementary operations in Section 1.1. For example, on setting in 1.1(19)

$$
a=u_{i}, \quad b=u_{j}, \quad u=u_{t}, \quad \gamma=\gamma_{t},
$$

and using $b_{t k}^{\text {abs }}, b_{t k}^{\text {rel }}$ from Table 1.2, the condition numbers $\lambda_{t}=\sigma_{t}^{i}, \rho_{t}^{i}$ in (18), (23) are specified as $\sigma, \rho$ in 1.1(19). The a posteriori condition numbers $\lambda_{t}=\sigma_{t}^{0}, \rho_{t}^{0}$ are obtained as $\sigma, \rho$ in 1.1(21). Also the bounds (19) are computed in this way.

When the graph of the algorithm is a tree and, additionally, $B$ a binary matrix, there exists for each node $k<t$ exactly one path to the node $t$ and the associated error effects are equal to 1 . From (21) we then infer

$$
L_{t k}=1, \quad k<t,
$$

and from (22) the condition numbers

$$
\lambda_{t}=\sum_{k=0}^{t} \alpha_{k}, \quad t=0, \ldots, n
$$


In view of the above, the numerical stability of evaluation algorithms for the condition numbers (18), (23) and the bounds (19) becomes of interest. The following, last theorem serves to answer this question. Note that $i_{t}, j_{t}$ denote the indices of the two operands in the arithmetic operations $F_{t}(u)=u_{i} \circ u_{j_{i}}$.

(26) Let the algorithm (A) possess the following properties: (a) all input data, intermediate, and final results $u_{0}, \ldots, u_{n}$ are positive numbers; (b) the algorithm consists only of input operations, additions, multiplications, and divisions. Then the recursion

$$
\begin{gathered}
\tau_{t}=\gamma_{t}, \quad F_{t} \in(\mathrm{F} 0), \\
\tau_{0}=\gamma_{0}, \quad \tau_{t}=\max \left(\tau_{i_{i}}, \tau_{j_{t}}\right)+\gamma_{t}, \quad F_{t}=+, \\
\tau_{t}=\tau_{i_{i}}+\tau_{j_{t}}+\gamma_{t}, \quad F_{t}=\times, /,
\end{gathered}
$$

determines a sequence of bounds for the relative a priori condition numbers of the algorithm, that is,

$$
\rho_{t} \leqslant \tau_{t}, \quad t=0, \ldots, n .
$$

Proof. The proposition is proved by finite induction with respect to $t$. For $t=0$, $\rho_{0}=\gamma_{0}=\tau_{0}$. Now assume that $\rho_{k} \leqslant \tau_{k}$ for $k=0, \ldots, t-1$. When $F_{t}$ is an input operation, $\rho_{t}=\gamma_{t}=\tau_{t}$. When $F_{t}$ is an addition, the solution of the linear relative a priori error equations satisfies the estimate

$$
\left|r_{t}\right| \leqslant\left|\frac{u_{i}}{u_{t}}\right|\left|r_{i}\right|+\left|\frac{u_{j}}{u_{t}}\right|\left|r_{j}\right|+\gamma_{t} \eta, \quad i=i_{t}, j=j_{t} .
$$

By assumption, $\left|r_{i}\right| \leqslant \tau_{i} \eta,\left|r_{j}\right| \leqslant \tau_{j} \eta$. As $u_{i}, u_{j}, u_{t}$ are positive, $\left|u_{i}\right|+\left|u_{j}\right|=\left|u_{t}\right|$, so that

$$
\frac{1}{\eta}\left|r_{t}\right| \leqslant\left|\frac{u_{i}}{u_{t}}\right| \tau_{i}+\left|\frac{u_{j}}{u_{t}}\right| \tau_{j}+\gamma_{t} \leqslant \max \left(\tau_{i}, \tau_{j}\right)+\gamma_{t}=\tau_{t} .
$$

Finally, $F_{t}=\times, /$ leads to the estimate

$$
\frac{1}{\eta}\left|r_{t}\right|<\left|r_{i}\right|+\left|r_{j}\right|+\gamma_{t} \leqslant \tau_{i}+\tau_{j}+\gamma_{t}=\tau_{t} .
$$

In this way, $\left|r_{t}\right| \leqslant \tau_{t} \eta$ for all $\left|e_{k}\right| \leqslant \gamma_{k} \eta, k=0, \ldots, n$, and by induction for all $t=0, \ldots, n$. In addition, from (16) for $f_{t}=e_{t} / \eta, L_{t}^{\text {relf }}=r_{t} / \eta$ one concludes $\lambda_{t}=\rho_{t}<\tau_{t}$.

Having determined the above sequence of bounds $\left(\tau_{t}\right)$, to each addition $F_{t}=+$ an index $m_{t} \in\left\{i_{t}, j_{t}\right\}$ can be assigned such that

$$
\tau_{m_{1}}=\max \left(\tau_{i_{i}}, \tau_{j_{i}}\right) \text {. }
$$

Let us further introduce the matrix $\left(\beta_{t k}\right)$ with the elements

$$
\begin{array}{ll}
\beta_{t k}=0, & F_{t} \in(\mathrm{F} 0), \\
\beta_{t k}=\delta_{k m_{t}}, & F_{t}=+, \\
\beta_{t k}=\delta_{k i_{t}}+\delta_{k j,}, & F_{t}=\times, /,
\end{array}
$$

for $k, t=0, \ldots, n$. Obviously, $\left(\beta_{t k}\right)$ is a binary matrix and the sequence of bounds $\left(\tau_{t}\right)$ is the solution of the linear system

$$
\tau_{t}-\sum_{k=0}^{t-1} \beta_{t k} \tau_{k}=\gamma_{t}, \quad t=0, \ldots, n .
$$


The graph of this system is obtained from the graph of the associated algorithm (A), named in Theorem (26), if to each node $t$ such that $F_{t}=+$ of the two paths $\overrightarrow{i_{t} t}, \overrightarrow{j_{t} t}$ only $\vec{m}_{t} t$ is kept and the other deleted. Denote by $N_{t k}$ the number of paths in this reduced graph from $k$ to $t$. Then, by (20), the following estimate and representation is established

$$
\rho_{t} \leqslant \gamma_{t}+\sum_{k=0}^{t-1} N_{t k} \gamma_{k}=\tau_{t}, \quad t=0, \ldots, n .
$$

3. A Survey of Examples and Applications. This section briefly surveys some typical examples and applications of the above error analysis.

3.1. The paper [18], in particular, studies the class of elementary one-step algorithms

$$
u_{0}=a, \quad u_{t}=b_{t} o_{t} u_{t-1}+c_{t}, \quad t=1, \ldots, n,
$$

where $o_{t} \in\{$ Nop, $\times, /, \backslash\}$. Special cases are the well-known algorithms for computing partial products $\left(o_{t}=\times, a=b_{0}, c_{t}=0\right)$, partial sums $\left(o_{t}=\right.$ Nop, $a=c_{0}$, $\left.b_{t}=1\right)$, solutions of inhomogeneous bidiagonal systems of linear equations $\left(o_{t}=\right.$ $\times)$, Horner's scheme for the evaluation of Taylor polynomials $\left(o_{t}=\times, a=c_{0}\right.$, $\left.b_{t}=z\right)$, Newton polynomials $\left(o_{t}=\times, a=c_{0}, b_{t}=z-z_{n-t}\right)$ and finite continued fractions $\left(o_{t}=/\right)$. The graph of the algorithm (1) is a tree so that by virtue of 2.3(23) the condition numbers can be determined recursively. For instance, the relative a priori condition numbers $\rho_{t}$ of computing $u_{t}$ from (1) satisfy the recursion

$$
\rho_{0}=\gamma_{0}^{a}, \quad \rho_{t}=\left|\frac{b_{t} o_{t} u_{t-1}}{u_{t}}\right|\left(\rho_{t-1}+\gamma_{t}^{b}+\gamma_{t}^{0}\right)+\left|\frac{c_{t}}{u_{t}}\right| \gamma_{t}^{c}+\gamma_{t}^{+}
$$

for $t=1, \ldots, n$. In addition, the paper [18] analyzes some further elementary algorithms and contains many numerical examples illustrating the error analysis.

3.2. The papers [16, Sections 4.3, 4.4], [19] deal with difference schemes

$$
u_{k}^{0}=u_{k} \quad(i=0), \quad u_{k}^{i}=u_{k}^{i-1}-u_{k-1}^{i-1},
$$

and the Neville-Aitken algorithm

$$
u_{k}^{0}=u_{k} \quad(i=0), \quad u_{k}^{i}=\frac{x-x_{k-i}}{x_{k}-x_{k-i}} u_{k}^{i-1}-\frac{x-x_{k}}{x_{k}-x_{k-i}} u_{k-1}^{i-1},
$$

$k=i, \ldots, m, i=1, \ldots, m$. The graphs of these algorithms are no longer tree-like but constitute an important tool in deriving the linear error equations and associated condition numbers. It is shown that also for the above algorithms the condition numbers can be obtained from simple recursion formulae. For difference schemes of a smooth function on equidistant meshes, using the condition numbers, a lower bound for the step-widths is obtained, called "critical step-width", which guarantees that at least the leading digit of the $m$ th order difference is significant. It is proved that Romberg extrapolations $\left(x_{k}=x_{0} / 4^{k}\right)$ are strongly stable. Further the condition numbers of the extrapolation algorithm (2) are determined for the systems of nodal points $x_{k}=x_{0} /(k+1)^{2}, x_{k}=x_{0} /(k+1)$ and applied to numerical examples. 
3.3. The paper [17] establishes the error analysis of numerically solving two linear equations in two unknowns,

$$
a x+b y=f, \quad c x+d y=g .
$$

The relative data and rounding condition numbers of computing the solutions $x, y$ by Cramer's rule and Gaussian elimination are determined. Two important results of the paper read: for a nonsingular linear system Cramer's rule is always well-conditioned or backward stable,

$$
\frac{\rho_{x}^{R}}{\rho_{x}^{D}}<2.5, \quad \frac{\rho_{y}^{R}}{\rho_{y}^{D}}<2.5
$$

Gaussian elimination is backward stable, and

$$
\frac{\rho_{x}^{R}}{\rho_{x}^{D}}<2.75, \quad \frac{\rho_{y}^{R}}{\rho_{y}^{D}}<2,
$$

provided that the system is properly pivoted such that $|b c|<|a d|$. The algorithms are analyzed further with respect to the behavior of the residuals of the computed solutions. It is shown that Gaussian elimination is, additionally, well-conditioned in this sense whereas Cramer's rule is not. It is proved that the relative condition numbers, the stability constants (2), (3), and the above pivotal strategy are invariant under scaling of the linear system.

3.4. In [20], [21], [22] the forward error analysis of Gaussian elimination and two-sided elimination of tridiagonal linear systems is presented. Both explicit representations and recursions of the absolute a priori data and rounding condition numbers $\sigma_{i}{ }^{D}, \sigma_{i}^{R}$ of the solutions $x_{i}, i=1, \ldots, n$, are derived. In addition, residual condition numbers $\tau_{j}{ }^{D}, \tau_{j}^{R}, j=1, \ldots, n$, are determined. When the tridiagonal coefficient matrix is an $M$-matrix or positive definite, Theorem [21, 2.3(21)] ensures both the backward stability and the residual stability of Gaussian elimination without pivoting for computing the solutions $x_{i}$ and proves the stability estimates

$$
\frac{\sigma_{i}^{R}}{\sigma_{i}^{D}} \leqslant 4, \quad \frac{\tau_{j}^{R}}{\tau_{j}^{D}} \leqslant 4, \quad i, j=1, \ldots, n .
$$

The paper [22] contains the corresponding forward error analysis of two-sided elimination. For every two-sided elimination-regular tridiagonal linear system, the computation of the solutions $x_{i}$ by two-sided elimination is well-conditioned or backward stable and

$$
\frac{\sigma_{i}^{R}}{\sigma_{i}^{D}} \leqslant 5.5, \quad i=1, \ldots, n .
$$

Babuška has proved the estimate $\sigma_{i}^{R} / \sigma_{i}^{D}<9$ in [2], using a backward error analysis. For the case $n=3$, Miller [12] has shown numerically that $\sigma_{2}^{R} / \sigma_{2}^{D}$ is about 5.5 so that our estimate (2) seems to be sharp for $n>3$. The general results of the forward error analysis are tested and illustrated by numerical examples in [20], [21], [22]. 
3.4. A forthcoming paper develops the error analysis of Gaussian elimination

$$
a_{i k}^{1}=a_{i k}, \quad a_{i k}^{t+1}=a_{i k}^{t}-\frac{a_{i t}^{t} a_{t k}^{t}}{a_{t t}^{t}},
$$

$i=t+1, \ldots, m, k=t+1, \ldots, n, t=1, \ldots, m-1$, for arbitrary rectangular matrices $A=\left(a_{i k}\right)_{i=1, \ldots, m ; k=1, \ldots, n}$ such that $a_{t t}^{t} \neq 0, t=1, \ldots, m-1$. The associated system of linear absolute a priori error equations reads

$$
s_{i k}^{1}=f_{i k}^{1}, \quad s_{i k}^{t+1}=s_{i k}^{t}+p_{i}^{t+1} s_{t k}^{t}+q_{k}^{t+1} s_{i t}^{t}+p_{i}^{t+1} q_{k}^{t+1} s_{t t}^{t}+f_{i k}^{t+1},
$$

where

$$
p_{i}^{t+1}=-\frac{a_{i t}^{t}}{a_{t t}^{t}}, \quad q_{k}^{t+1}=-\frac{a_{t k}^{t}}{a_{t t}^{t}},
$$

$f_{i k}^{1}$ are the absolute errors of the coefficients $a_{i k}$, and

$$
f_{i k}^{t}=a_{i k}^{t} e_{t i k}^{-}-p_{i}^{t} q_{k}^{t} a_{t-1, t-1}^{t-1}\left(e_{t i k}^{\times}+e_{t i k}^{\cdot / \cdot}\right), \quad t=2, \ldots, m .
$$

An explicit representation of the solution $s_{i k}^{t}$ in terms of the $f_{i k}^{t}$ is obtained that immediately yields associated condition numbers $\sigma_{i k}^{t}$.

The error analysis of this important algorithm is applied to forward elimination of a system of $m$ linear equations with $n-m$ right-hand sides, back substitution or solving a triangular system of linear equations, solving an inhomogeneous linear system, and computing the inverse of a nonsingular square matrix.

This forward error analysis differs significantly from Wilkinson's backward error analysis. Forward error analysis compares the numerical results obtained under data perturbations or rounding errors of a floating-point arithmetic directly with the exact results and establishes optimal bounds of the possible errors, whereas backward error analysis primarily estimates the residuals of approximate solutions and subsequently obtains error estimates of solution vectors in suitable norms by means of condition numbers of the coefficient matrix. This procedure may overestimate the actual error considerably. Moreover, the forward error analysis uses a 'finer topology': the error estimates bound the components of the error vectors and do not use norms; the condition numbers and stability constants depend on the solutions and thus yield pointwise, not uniform, estimates. Finally, our stability constants and relative data and rounding condition numbers are invariant with respect to scaling of the linear system.

Acknowledgement. The author acknowledges valuable comments of the referees.

Fachbereich Mathematik

University of Frankfurt

Robert-Mayer Strasse 10

D-6000 Frankfurt, West Germany

1. I. BabušKa, Numerical Stability in Numerical Analysis, Proc. IFIP-Congress 1968, Amsterdam, North-Holland, Amsterdam, 1969, pp. 11-23.

2. I. BABUŠKA, "Numerical stability in problems of linear algebra," SIAM J. Numer. Anal., v. 9, 1972, pp. 53-77.

3. I. Babuška, M. Prager \& E. Vitasek, Numerical Processes in Differential Equations, Wiley, New York, 1966.

4. F. L. BAUER, "Genauigkeitsfragen bei der Lösung linearer Gleichungssysteme," Z. Angew. Math. Mech., v. 46, 1966, pp. 409-421. 

87-96.

5. F. L. BAUER, "Computational graphs and rounding error," SIAM J. Numer. Anal., v. 11, 1974, pp.

6. F. L. BAUER ET AL., Moderne Rechenanlagen, Chap. 3, Teubner, Stuttgart, 1964.

7. W. S. Brown, A Realistic Model of Floating-Point Computation, Proc. Sympos. Mathematical Software III, Madison, 1977 (J. R. Rice, Ed.), Academic Press, New York, 1977, pp. 343-360.

8. P. Henrici, Elements of Numerical Analysis, Chap. 16, Wiley, New York, 1964.

9. J. Larson \& A. SAMrh, "Efficient calculation of the effects of roundoff errors," ACM Trans. Math. Software, v. 4, 1978, pp. 228-236.

10. S. LinnainmaA, "Taylor expansion of the accumulated rounding error," BIT, v. 16, 1976, pp. 146-160.

11. D. D. MCCracken \& W. S. Dorn, Numerical Methods and Fortran Programming, Wiley, New York, 1964.

12. W. Miller, "Software for roundoff analysis," ACM Trans. Math. Software, v. 1, 1975, pp. 108-128.

13. W. Miller, "Computer search for numerical instability," J. Assoc. Comput. Mach., v. 22, 1975, pp. 512-521.

14. W. Miller \& D. SPOONER, "Software for roundoff analysis, II," ACM Trans. Math. Software, v. 4, 1978, pp. 369-387.

15. J. R. RiCE, "A theory of condition," SIAM J. Numer. Anal., v. 3, 1966, pp. 287-310.

16. F. STUMme, Fehleranalyse numerischer Algorithmen, Lecture Notes, Univ. of Frankfurt, 1978.

17. F. STUMmeL, "Rounding errors in numerical solutions of two linear equations in two unknowns," Preprint, 1980.

18. F. STUMme, Rounding Error Analysis of Elementary Numerical Algorithms, Proc. Conf. Fundamentals of Numerical Computation, Berlin 1979 (G. Alefeld \& R. D. Grigorieff, Eds.), Computing, Suppl. 2, 1980, pp. 169-195.

19. F. STUMMEL, "Rounding error anlaysis of difference and extrapolation schemes." (To appear.)

20. F. STUMmEL, Rounding Error in Gaussian Elimination of Tridiagonal Linear Systems, Survey of Results, Proc. Internat. Sympos. 'Interval Mathematics 1980', Freiburg (K. Nickel, Ed.), Academic Press, New York, 1980, pp. 223-245.

21. F. STUMmEL, "Rounding error in Gaussian elimination of tridiagonal linear systems. I," SIAM J. Numer. Anal. (Submitted.)

22. F. STUMme, "Rounding error in Gaussian elimination of tridiagonal linear systems. II," Linear Algebra Appl. (Submitted.)

23. F. STUMMEL, "Forward error analysis of Gaussian elimination." (To appear.)

24. M. TIENARI, "On some topological properties of numerical algorithms," BIT, v. 12, 1972, pp. 409-433.

25. J. H. Wilkinson, Rounding Errors in Algebraic Processes, Prentice-Hall, Englewood Cliffs, N.J., 1963. 